\title{
A Dynamic Contact Problem with History-Dependent Operators
}

\author{
Justyna Ogorzały ${ }^{1}$
}

Received: 12 May 2015 / Published online: 15 January 2016

(C) The Author(s) 2016. This article is published with open access at Springerlink.com

\begin{abstract}
In this paper we present results on existence, uniqueness and convergence of solutions to the Cauchy problem for abstract first order evolutionary inclusion which contains two operators depending on the history of the solution. These results are applicable to a dynamic contact problem for viscoelastic materials with a normal compliance contact condition with memory and a friction law in which the friction bound depends on the magnitude of the tangential displacement. The proofs are based on recent results for hemivariational inequalities and a fixed point argument.
\end{abstract}

Keywords Viscoelastic material · History-dependent operator - Clarke subdifferential · Multifunction · Dynamical process · Friction

Mathematics Subject Classification 35Q74 - 47J20 $\cdot 74 \mathrm{M} 10 \cdot 74 \mathrm{M} 15 \cdot 74 \mathrm{H} 20 \cdot 74 \mathrm{H} 25$

\section{Introduction}

The subject of our research is a contact model that involves history-dependent operators. This model describes the dynamic frictional contact between a viscoelastic body and a foundation. We consider the contact condition with a normal compliance and a memory effect of the foundation. The research that we present in this paper concerns the study on existence and uniqueness of solutions to the hemivariational inequalities with history-dependent operators, and the investigation on the behaviour of solutions to such inequalities with respect to perturbations of operators and functions.

Research supported by the Marie Curie International Research Staff Exchange Scheme Fellowship within the 7th European Community Framework Programme under Grant Agreement No. 295118 and the National Science Center of Poland under the Maestro Project no. DEC-2012/06/A/ST1/00262.

J. Ogorzały

justyna.ogorzaly@gmail.com

1 Institute of Mathematics, Jagiellonian University in Krakow, ul. prof. S. Lojasiewicza 6, 30-348 Kraków, Poland 
We note that the history-dependent operators have been considered for quasistatic and evolutionary contact problems by several authors, for example, by Sofonea et al. in [8, 21, 26-28] and [29], Migórski et al. in [5, 15, 19] and [20], Ogorzały in [23], Yao et al. in [31], and Zhu in [33]. The normal compliance contact condition was introduced in $[13,14,22]$ and it was used in many papers, see, e.g., [3, 9, 25] and [29]. In [28], Sofonea and Patrulescu considered a contact condition which describes a foundation that is rigid if the penetration reaches a critical bound. Moreover, their model takes into account the memory effect of the foundation. In turn, Farcas et al. in [8] studied the model which describes the quasistatic contact between a viscoplastic body and a foundation. The authors used a contact condition which involves both a normal compliance, a unilateral constraint and a memory effect on the foundation. They proved that the problem under consideration has a unique solution. In [25], Sofonea et al., the behaviour of the solutions of variational inequalities with history-dependent operator is investigated. In our paper we use the contact condition with the normal compliance and memory effect which was introduced in [5] in the study of a quasistatic contact problem. In contrast to [5], our model is dynamic and we assume that the magnitude of the friction force is bounded by a function, the so-called friction bound. We also mention that various classes of hemivariational inequalities for contact problems with slip-dependent friction have been studied, e.g., in [17] and [18].

In the present paper, we start with the study of an abstract evolutionary inclusion of the first order with a history-dependent operator. We prove results on existence and uniqueness of solution and a result on the convergence of solution of the inclusion with historydependent operators with respect to perturbations in the data. The existence result is obtained by a technique used by Migórski and Kulig in [20] who studied second order subdifferential inclusions with a Volterra-type operator. The unique solvability of the inclusion is proved by a standard fixed point argument similar to those used in many papers, for instance in $[15,19-21]$ and [23]. Furthermore, we note that the abstract convergence result of Theorem 13 is based on arguments and assumptions similar to those exploited, for instance in $[2,15,25]$ and [27]. In the second part of the paper we analyze a mathematical model of a contact problem for viscoelastic materials with history-dependent operators and a slipdependent friction. We provide its variational formulation and deliver results on existence, uniqueness and convergence of solutions.

There are two main novel features of this paper. First novelty consists in the more general form of the friction law. We study a contact model which involves the friction condition with the friction bound depending on the magnitude of the tangential displacement. Such dependence is met in several models of Contact Mechanics, for example, in $[1,10-12,16,18]$ and [30]. In the analysis of frictional processes, such as modelling of motions of tectonic plates and earthquakes, it is necessary to consider the friction bound which varies with the magnitude of the tangential displacement. In the aformentioned situations, the constant friction bound is inadequate to model the dynamic frictional contact. Another novel feature of this paper is the analysis of the dynamics. In contrast to other contributions in the field, cf., e.g., [1, 12, 17, 27] and [31], we treat a dynamic contact problem for which the mathematical techniques are less developed than for quasistatic evolutionary models. We underline that there are no results on existence, uniqueness and convergence of solutions to the dynamic hemivariational inequality in Problem 17, which models the contact problem under consideration.

The rest of paper is structured as follows. In Sect. 2, we recall some preliminary material. In Sect. 3, we state and prove our abstract existence, uniqueness and convergence results. In Sect. 4 , we study the dynamic contact problem for which we use the results obtained for the abstract inclusion. 


\section{Preliminaries}

In this section we present a material which is helpful in the rest of the paper. Let $X$ be a Banach space with norm $\|\cdot\|_{X}$ and let $X^{*}$ be its dual. The notation $\langle\cdot, \cdot\rangle_{X^{*} \times X}$ stands for the duality pairing between $X^{*}$ and $X$, and $2^{X^{*}}$ represents the collection of all subsets of $X$. We denote by $\mathcal{L}(X, Y)$ the space of linear and bounded operators defined on a Banach space $X$ with values in a Banach space $Y$ with the usual norm $\|\cdot\|_{\mathcal{L}(X, Y)}$. For a set $U \subset X$, we define $\|U\|_{X}=\sup \left\{\|u\|_{X} \mid u \in U\right\}$.

In the paper we use the concepts of the generalized directional derivative and the Clarke subdifferential, see, for example, [6, 7] and [21].

Definition 1 The generalized directional derivative (in the sense of Clarke) of a locally Lipschitz function $\varphi: X \longrightarrow \mathbb{R}$ at the point $x \in X$ in the direction $v \in X$, denoted $\varphi^{0}(x ; v)$ is defined by

$$
\varphi^{0}(x ; v)=\limsup _{y \rightarrow x, \lambda \downarrow 0} \frac{\varphi(y+\lambda v)-\varphi(y)}{\lambda} .
$$

Definition 2 Let $\varphi: X \rightarrow \mathbb{R}$ be a locally Lipschitz function. The Clarke generalized gradient (subdifferential) of $\varphi$ at $x \in X$, denoted $\partial \varphi(x)$, is the subset of a dual space $X^{*}$ defined by

$$
\partial \varphi(x)=\left\{\zeta \in X^{*} \mid \varphi^{0}(x ; v) \geqslant\langle\zeta, v\rangle_{X^{*} \times X} \text { for all } v \in X\right\}
$$

We have the following definition of regularity in sense of Clarke (see, for instance, [6]).

Definition 3 A locally Lipschitz function $\varphi: X \longrightarrow \mathbb{R}$ is called regular at $x \in X$, if for all $v \in X$, the one-sided directional derivative $\varphi^{\prime}(x, v)$ exists and it satisfies $\varphi^{0}(x ; v)=\varphi^{\prime}(x, v)$ for all $v \in X$.

The proof of the following result can be found in Theorem 2.3.10 in [6].

Proposition 4 Let $X$ and $Y$ be Banach spaces, $L \in \mathcal{L}(Y, X)$ and let $\varphi: X \longrightarrow \mathbb{R}$ be a locally Lipschitz function. Then

(i) $(\varphi \circ L)^{0}(x ; z) \leqslant \varphi^{0}(L x ; L z)$ for $x, z \in Y$,

(ii) $\partial(\varphi \circ L)(x) \subseteq L^{*} \partial \varphi(L x)$ for $x \in Y$,

where $L^{*} \in \mathcal{L}\left(X^{*}, Y^{*}\right)$ denotes the adjoint operator to $L$. If, in addition, either $\varphi$ or $-\varphi$ is regular, then (i) and (ii) hold with the corresponding equalities.

We recall the result which is a consequence of the Banach contraction principle (for the proof see, for instance, [15]).

Lemma 5 Let $X$ be a Banach space with a norm $\|\cdot\|_{X}$ and $T>0$. Let $\Lambda: L^{2}(0, T ; X) \longrightarrow$ $L^{2}(0, T ; X)$ be an operator such that

$$
\left\|\left(\Lambda \eta_{1}\right)(t)-\left(\Lambda \eta_{2}\right)(t)\right\|_{X}^{2} \leqslant c \int_{0}^{t}\left\|\eta_{1}(s)-\eta_{2}(s)\right\|_{X}^{2} d s
$$

for every $\eta_{1}, \eta_{2} \in L^{2}(0, T ; X)$, a.e. $t \in(0, T)$ with a constant $c>0$. Then $\Lambda$ has a unique fixed point in $L^{2}(0, T ; X)$, i.e., there exists a unique $\eta^{*} \in L^{2}(0, T ; X)$ such that $\Lambda \eta^{*}=\eta^{*}$. 
Here and subsequently $c$ denotes a positive constant which value can change from line to line.

Finally, we recall an existence and uniqueness result for the evolutionary inclusion of first order. We assume that $V$ and $Z$ are separable and reflexive Banach spaces with the duals $V^{*}$ and $Z^{*}$, respectively. Let $H$ denote a separable Hilbert space and we identify $H$ with its dual. We suppose that $V \subset H \subset V^{*}$ and $Z \subset H \subset Z^{*}$ are Gelfand triples of spaces where all embeddings are continuous, dense and compact (see, for instance, Chap. 23.4 of [32], Chap. 3.4 of [7]). Moreover, for $0<T<\infty$, we introduce the following spaces $\mathcal{V}=L^{2}(0, T ; V)$ and $\mathcal{W}=\left\{v \in \mathcal{V} \mid v^{\prime} \in \mathcal{V}^{*}\right\}$. The first order evolutionary inclusion reads as follows.

Problem 6 Find $u \in \mathcal{W}$ such that

$$
\left\{\begin{array}{l}
u^{\prime}(t)+A(t, u(t))+G(t, u(t)) \ni f(t) \quad \text { a.e. } t \in(0, T), \\
u(0)=v_{0} .
\end{array}\right.
$$

We assume the following hypotheses on the data of Problem 6.

$A:(0, T) \times V \longrightarrow V^{*}$ is such that

(a) $A(\cdot, u)$ is measurable on $(0, T)$ for all $u \in V$,

(b) $A(t, \cdot)$ is strongly monotone, i.e., there exists $m_{A}>0$ such that $\left\langle A\left(t, u_{1}\right)-A\left(t, u_{2}\right), u_{1}-u_{2}\right\rangle_{V^{*} \times V} \geqslant m_{A}\left\|u_{1}-u_{2}\right\|_{V}^{2}$

for all $u_{1}, u_{2} \in V$ and a.e. $t \in(0, T)$,

(c) $A(t, \cdot)$ is hemicontinuous, i.e., for all $u, v, w \in V$, the function $t \longmapsto\langle A(u+t v), w\rangle_{V^{*} \times V}$ is continuous on [0,1],

(d) $\|A(t, u)\|_{V^{*}} \leqslant a_{0}(t)+a_{1}\|u\|_{V}$ for all $u \in V$, a.e. $t \in(0, T)$ with $a_{0} \in L^{2}(0, T)$ and $a_{0}, a_{1} \geqslant 0$,

(e) $A(t, 0)=0$ for a.e. $t \in(0, T)$.

$G:(0, T) \times V \longrightarrow 2^{Z^{*}}$ has nonempty, closed, convex values and

(a) $G(\cdot, v)$ is measurable on $(0, T)$ for all $v \in V$,

(b) $G(t, \cdot)$ is upper semicontinuous from $V$ into $w-Z^{*}$ for a.e. $t \in(0, T)$, where $V$ is endowed with $Z$-topology,

(c) $\|G(t, v)\|_{Z^{*}} \leqslant \tilde{d}_{0}(t)+\tilde{d}_{1}\|v\|_{V}$ for all $v \in V$, a.e. $t \in(0, T)$ with $\tilde{d}_{0} \in L^{2}(0, T)$ and $\tilde{d}_{0}, \tilde{d}_{1} \geqslant 0$,

(d) $\left\langle\zeta_{1}-\zeta_{2}, v_{1}-v_{2}\right\rangle_{Z^{*} \times Z} \geqslant-m_{2}\left\|v_{1}-v_{2}\right\|_{V}^{2}$ for all $\zeta_{i} \in G\left(t, v_{i}\right), v_{i} \in V$, $i=1$, 2, a.e. $t \in(0, T)$ with $m_{2} \geqslant 0$.

(a) $f \in \mathcal{V}^{*}$ and $v_{0} \in V$,

(b) $m_{A}>m_{2}$,

(c) $m_{A}>2 \sqrt{3} c_{\mathrm{e}} \tilde{d}_{1}$, where $c_{\mathrm{e}}>0$ is the embedding constant of $V$ into $Z$, i.e., $\|\cdot\|_{Z} \leqslant c_{\mathrm{e}}\|\cdot\|_{V}$. 
Theorem 7 Under assumptions (1)-(3), Problem 6 has a unique solution.

Proof It follows the lines of proof of Theorem 3 in [15], cf. Sects. 3.2.2 and 3.2.3 in [15].

\section{History-Dependent Subdifferential Inclusion}

In this section we study the Cauchy problem for a subdifferential inclusion with historydependent operators. We provide an existence and uniqueness result and a theorem on the dependence of solution on the data. Here, we use the spaces $V, Z, H, \mathcal{V}$ and $\mathcal{W}$ which are defined in Sect. 2. Additionally, we denote by $C(0, T ; H)$ the space of continuous functions from $[0, T]$ into $H$. We consider the following abstract inclusion.

Problem 8 Find $u \in \mathcal{W}$ such that

$$
\left\{\begin{array}{l}
u^{\prime}(t)+A(t, u(t))+(\mathcal{S} u)(t)+F(t,(\mathcal{R} u)(t), u(t)) \ni f(t) \quad \text { for a.e. } t \in(0, T) \\
u(0)=v_{0}
\end{array}\right.
$$

We admit the following definition.

Definition 9 A function $u \in \mathcal{W}$ is a solution of Problem 8 if and only if there exists $\zeta \in$ $L^{2}\left(0, T ; Z^{*}\right)$ such that

$$
\begin{cases}u^{\prime}(t)+A(t, u(t))+(\mathcal{S} u)(t)+\zeta(t)=f(t) & \text { for a.e. } t \in(0, T) \\ \zeta(t) \in F(t,(\mathcal{R} u)(t), u(t)) & \text { for a.e. } t \in(0, T) \\ u(0)=v_{0} & \end{cases}
$$

For operators $\mathcal{R}$ and $\mathcal{S}$, we need the following hypotheses.

$\mathcal{R}: \mathcal{V} \longrightarrow \mathcal{V}$ is such that

$$
\left\|\left(\mathcal{R} u_{1}\right)(t)-\left(\mathcal{R} u_{2}\right)(t)\right\|_{V} \leqslant L_{\mathcal{R}} \int_{0}^{t}\left\|u_{1}(s)-u_{2}(s)\right\|_{V} d s
$$

for $u_{1}, u_{2} \in \mathcal{V}$, a.e. $t \in(0, T)$ with $L_{\mathcal{R}}>0$.

$\mathcal{S}: \mathcal{V} \longrightarrow \mathcal{V}^{*}$ is such that

$$
\left\|\left(\mathcal{S} u_{1}\right)(t)-\left(\mathcal{S} u_{2}\right)(t)\right\|_{V^{*}} \leqslant L_{\mathcal{S}} \int_{0}^{t}\left\|u_{1}(s)-u_{2}(s)\right\|_{V} d s
$$

for $u_{1}, u_{2} \in \mathcal{V}$, a.e. $t \in(0, T)$ with $L_{\mathcal{S}}>0$.

In the case of operators (5) and (6), the values $(\mathcal{R} v)(t)$ and $(\mathcal{S} v)(t)$, respectively, at the moment $t$ depend on the history of the value of $v$ at the moment $0 \leqslant s \leqslant t$. For this reason these operators are called the history-dependent operators. Some examples of the historydependent operators can be found, for instance, in [27]. 
For the multifunction $F$ in Problem 8, we need the following assumption.

$F:(0, T) \times V \times V \longrightarrow 2^{Z^{*}}$ has nonempty, closed, convex values and

(a) $F(\cdot, u, v)$ is measurable on $(0, T)$ for all $u, v \in V$,

(b) $F(t, \cdot, \cdot)$ is upper semicontinuous from $V \times V$ into $w-Z^{*}$ for a.e. $t \in(0, T)$,

(c) $\|F(t, u, v)\|_{Z^{*}} \leqslant d_{0}(t)+d_{1}\|u\|_{V}+d_{2}\|v\|_{V}$ for all $u, v \in V$, a.e. $t \in(0, T)$, with $d_{0} \in L^{2}(0, T)$ and $d_{0}, d_{1}, d_{2} \geqslant 0$,

(d) $\left\langle\zeta_{1}-\zeta_{2}, v_{1}-v_{2}\right\rangle_{Z^{*} \times Z} \geqslant-m_{F 1}\left\|v_{1}-v_{2}\right\|_{V}^{2}-m_{F 2}\left\|v_{1}-v_{2}\right\|_{V}\left\|u_{1}-u_{2}\right\|_{V}$ for all $\zeta_{i} \in F\left(t, u_{i}, v_{i}\right), u_{i}, v_{i} \in V, i=1,2$, a.e. $t \in(0, T)$ with $m_{F 1}, m_{F 2} \geqslant 0$.

Additionally, we need the following smallness conditions

$\left.\begin{array}{l}\text { (a) } m_{A}>2 \sqrt{3} c_{\mathrm{e}} d_{2} \\ \text { (b) } m_{A}>m_{F 1}\end{array}\right\}$

Theorem 10 Assume that (1), (3)(a) and (5)-(8) hold. Then Problem 8 has a unique solution.

Proof Let $(\eta, \xi) \in \mathcal{V}^{*} \times \mathcal{V}$ be given. We consider the following auxiliary problem.

Problem 11 Find $u_{\eta \xi} \in \mathcal{W}$ such that

$$
\left\{\begin{array}{l}
u_{\eta \xi}^{\prime}(t)+A\left(t, u_{\eta \xi}(t)\right)+F\left(t, \xi(t), u_{\eta \xi}(t)\right) \ni f(t)-\eta(t) \quad \text { for a.e. } t \in(0, T), \\
u_{\eta \xi}(0)=v_{0} .
\end{array}\right.
$$

We establish the existence of a unique solution to Problem 11 by using Theorem 7. Let $G_{\xi}:(0, T) \times V \longrightarrow 2^{Z^{*}}$ be defined by $G_{\xi}(t, v)=F(t, \xi(t), v)$ for all $v \in V$ and a.e. $t \in(0, T)$. It easy to see that $G_{\xi}(\cdot, v)$ is measurable on $(0, T)$ for all $v \in V$ and $G_{\xi}(t, \cdot)$ is upper semicontinuous from $V$ into $w-Z^{*}$ for a.e. $t \in(0, T)$. From (7)(c), we infer that $\left\|G_{\xi}(t, v)\right\|_{Z^{*}}=\|F(t, \xi(t), v)\|_{Z^{*}} \leqslant d_{0}(t)+d_{1}\|\xi(t)\|_{V}+d_{2}\|v\|_{V}$. Hence $G_{\xi}$ satisfies hypothesis $(2)(\mathrm{c})$ with $\tilde{d}_{0}(t)=d_{0}(t)+d_{1}\|\xi(t)\|_{V}, \tilde{d}_{0} \in L^{2}(0, T)$ and $\tilde{d}_{1}=d_{2}$. Finally, from (7)(d), we have

$$
\begin{aligned}
& \left\langle G_{\xi}\left(t, v_{1}\right)-G_{\xi}\left(t, v_{2}\right), v_{1}-v_{2}\right\rangle_{Z^{*} \times Z}=\left\langle F\left(t, \xi(t), v_{1}\right)-F\left(t, \xi(t), v_{2}\right), v_{1}-v_{2}\right\rangle_{Z^{*} \times Z} \\
& \geqslant-m_{F 1}\left\|v_{1}-v_{2}\right\|_{V}^{2} \quad \text { for all } v_{1}, v_{2} \in V, \text { a.e. } t \in(0, T) .
\end{aligned}
$$

Hence, we deduce that the operator $G_{\xi}$ satisfies condition (2)(d) with $m_{2}=m_{F 1}$. Since $d_{2}=\tilde{d}_{1}$, condition (8)(a) implies (3)(c). Using the fact that $m_{2}=m_{F 1}$, from (8)(b), we deduce (3)(b). Therefore, the hypotheses of Theorem 7 are verified. From this theorem, we infer that Problem 11 has a unique solution $u_{\eta \xi} \in \mathcal{W}$.

Next, we will establish an inequality which shows the dependence of the solution $u_{\eta \xi} \in$ $\mathcal{W}$ of Problem 11 on $\eta$ and $\xi$. Let $u_{\eta_{i} \xi_{i}}=u_{i} \in \mathcal{W}$ be the unique solution to Problem 11 corresponding to $\left(\eta_{i}, \xi_{i}\right) \in \mathcal{V}^{*} \times \mathcal{V}$ for $i=1,2$. Then, there exists $\zeta_{i} \in L^{2}\left(0, T ; Z^{*}\right)$ such that $\zeta_{i}(t) \in F\left(t, \xi_{i}(t), u_{i}(t)\right)$ for a.e. $t \in(0, T), i=1,2$, and we have

$$
u_{1}^{\prime}(t)+A\left(t, u_{1}(t)\right)+\eta_{1}(t)+\zeta_{1}(t)=f(t)
$$




$$
u_{2}^{\prime}(t)+A\left(t, u_{2}(t)\right)+\eta_{2}(t)+\zeta_{2}(t)=f(t)
$$

for a.e. $t \in(0, T)$. Multiplying Eq. (10) by $u_{2}(t)-u_{1}(t)$ and Eq. (11) by $u_{1}(t)-u_{2}(t)$, and adding the results, we obtain

$$
\begin{gathered}
\left\langle u_{1}^{\prime}(t)-u_{2}^{\prime}(t), u_{2}(t)-u_{1}(t)\right\rangle_{V^{*} \times V}+\left\langle A\left(t, u_{1}(t)\right)-A\left(t, u_{2}(t)\right), u_{2}(t)-u_{1}(t)\right\rangle_{V^{*} \times V} \\
+\left\langle\eta_{1}(t)-\eta_{2}(t), u_{2}(t)-u_{1}(t)\right\rangle_{V^{*} \times V}+\left\langle\zeta_{1}(t)-\zeta_{2}(t), u_{2}(t)-u_{1}(t)\right\rangle_{Z^{*} \times Z}=0 .
\end{gathered}
$$

Integrating the above inequality over $(0, t)$, for all $t \in[0, T]$, and next using the integration by parts formula, the Cauchy-Schwartz inequality, conditions (1)(b) and (7)(d), we have

$$
\begin{aligned}
& \frac{1}{2}\left\|u_{1}(t)-u_{2}(t)\right\|_{H}^{2}+m_{A} \int_{0}^{t}\left\|u_{1}(s)-u_{2}(s)\right\|_{V}^{2} d s-m_{F 1} \int_{0}^{t}\left\|u_{1}(s)-u_{2}(s)\right\|_{V}^{2} d s \\
& \leqslant m_{F 2} \int_{0}^{t}\left\|\xi_{1}(t)-\xi_{2}(t)\right\|_{V}\left\|u_{1}(s)-u_{2}(s)\right\|_{V} d s \\
& \quad+\int_{0}^{t}\left\|\eta_{1}(s)-\eta_{2}(s)\right\|_{V^{*}}\left\|u_{1}(s)-u_{2}(s)\right\|_{V} d s
\end{aligned}
$$

Omitting the first term on the left hand side, using condition (8)(b) and the Hölder inequality, we obtain

$$
\left\|u_{1}-u_{2}\right\|_{L^{2}(0, t ; V)} \leqslant c\left(\left\|\eta_{1}-\eta_{2}\right\|_{L^{2}\left(0, t ; V^{*}\right)}+\left\|\xi_{1}-\xi_{2}\right\|_{L^{2}(0, t ; V)}\right) \quad \text { for a.e. } t \in(0, T) .
$$

Next, we define the operator $\Lambda: L^{2}\left(0, T ; V^{*} \times V\right) \longrightarrow L^{2}\left(0, T ; V^{*} \times V\right)$ by

$$
\Lambda(\eta, \xi)=\left(\mathcal{S} u_{\eta \xi}, \mathcal{R} u_{\eta \xi}\right) \quad \text { for }(\eta, \xi) \in L^{2}\left(0, T ; V^{*} \times V\right),
$$

where $u_{\eta \xi} \in \mathcal{W}$ denotes the unique solution to Problem 11 corresponding to $(\eta, \xi)$. Then, from (5) and (6), we get

$$
\begin{aligned}
& \left\|\Lambda\left(\eta_{1}, \xi_{1}\right)(t)-\Lambda\left(\eta_{2}, \xi_{2}\right)(t)\right\|_{V^{*} \times V}^{2}=\left\|\left(\mathcal{S} u_{1}\right)(t)-\left(\mathcal{S} u_{2}\right)(t)\right\|_{V^{*}}^{2}+\left\|\left(\mathcal{R} u_{1}\right)(t)-\left(\mathcal{R} u_{2}\right)(t)\right\|_{V}^{2} \\
& \leqslant\left(L_{\mathcal{S}} \int_{0}^{t}\left\|u_{1}(s)-u_{2}(s)\right\|_{V} d s\right)^{2}+\left(L_{\mathcal{R}} \int_{0}^{t}\left\|u_{1}(s)-u_{2}(s)\right\|_{V} d s\right)^{2}
\end{aligned}
$$

for a.e. $t \in(0, T)$. Using the Hölder inequality, we see that

$$
\left\|\Lambda\left(\eta_{1}, \xi_{1}\right)(t)-\Lambda\left(\eta_{2}, \xi_{2}\right)(t)\right\|_{V^{*} \times V}^{2} \leqslant\left(L_{\mathcal{S}}^{2}+L_{\mathcal{R}}^{2}\right) T \int_{0}^{t}\left\|u_{1}(s)-u_{2}(s)\right\|_{V}^{2} d s
$$

for a.e. $t \in(0, T)$. Combining the latter with (12), we obtain the following inequality

$$
\left\|\Lambda\left(\eta_{1}, \xi_{1}\right)(t)-\Lambda\left(\eta_{2}, \xi_{2}\right)(t)\right\|_{V^{*} \times V}^{2} \leqslant c\left(\left\|\eta_{1}-\eta_{2}\right\|_{L^{2}\left(0, t ; V^{*}\right)}^{2}+\left\|\xi_{1}-\xi_{2}\right\|_{L^{2}(0, t ; V)}^{2}\right) .
$$

for a.e. $t \in(0, T)$. Based on Lemma 5 and estimate (13), we conclude that the operator $\Lambda$ has a unique fixed point $\left(\eta^{*}, \xi^{*}\right) \in \mathcal{V}^{*} \times \mathcal{V}$. This implies that $u_{\eta^{*} \xi^{*}} \in \mathcal{W}$ is a solution of Problem 8, which concludes the existence part of the theorem.

The uniqueness of solution to Problem 8 is a consequence of the uniqueness of the fixed point of $\Lambda$. Namely, let $u \in \mathcal{W}$ be a solution to Problem 8 and define the element $(\eta, \xi) \in$ $\mathcal{V}^{*} \times \mathcal{V}$ by $(\eta, \xi)=(\mathcal{S} u, \mathcal{R} u)$. It follows that $u$ is the solution to Problem 11 and, by the 
uniqueness of solutions to Problem 11, we obtain that $u=u_{\eta \xi}$. This implies that $\Lambda(\eta, \xi)=$ $(\eta, \xi)$. By the uniqueness of the fixed point of $\Lambda$, we have $(\eta, \xi)=\left(\eta^{*}, \xi^{*}\right)$, so $u=u_{\eta^{*} \xi^{*}}$. This completes the proof of the theorem.

Now, we study the dependence of solution of the history-dependent subdifferential inclusion (4) with respect to the perturbations of the operators $A, \mathcal{R}, \mathcal{S}$ and the function $f$. For every $\rho>0$, let $A_{\rho}, \mathcal{R}_{\rho}, \mathcal{S}_{\rho}, f_{\rho}$ and $u_{0 \rho}$ be perturbations of $A, \mathcal{R}, \mathcal{S}, f$ and $u_{0}$, respectively. We introduce the following hypotheses.

(a) the operators $A, A_{\rho}:(0, T) \times V \longrightarrow V^{*}$ satisfy (1) and $A_{\rho}(t, v) \longrightarrow A(t, v)$ in $V^{*}$ for all $v \in V$, a.e. $t \in(0, T)$, as $\rho \longrightarrow 0$,

(b) the operators $\mathcal{S}, \mathcal{S}_{\rho}: \mathcal{V} \longrightarrow \mathcal{V}^{*}$ satisfy (6) with $L_{\mathcal{S}}>0$ and $L_{\mathcal{S}_{\rho}}>0$ for all $\rho>0$, respectively and $L_{\mathcal{S}_{\rho}} \leqslant L_{1}$ for all $\rho>0$,

(c) there exists $k_{1}: \mathbb{R}_{+} \longrightarrow \mathbb{R}_{+}$and $g_{1}: \mathcal{V} \longrightarrow \mathbb{R}_{+}$such that $\left\|\left(\mathcal{S}_{\rho} v\right)(t)-(\mathcal{S} v)(t)\right\|_{V^{*}} \leqslant k_{1}(\rho) g_{1}(v)$ for all $v \in \mathcal{V}$, a.e. $t \in(0, T)$ and $\lim _{\rho \rightarrow 0} k_{1}(\rho)=0$,

(d) the operators $\mathcal{R}, \mathcal{R}_{\rho}: \mathcal{V} \longrightarrow \mathcal{V}$ satisfy (5) with $L_{\mathcal{R}}>0$ and $L_{\mathcal{R}_{\rho}}>0$ for all $\rho>0$, respectively and $L_{\mathcal{R}_{\rho}} \leqslant L_{2}$ for all $\rho>0$,

(e) there exists $k_{2}: \mathbb{R}_{+} \longrightarrow \mathbb{R}_{+}$and $g_{2}: \mathcal{V} \longrightarrow \mathbb{R}_{+}$such that $\left\|\left(\mathcal{R}_{\rho} v\right)(t)-(\mathcal{R} v)(t)\right\|_{V} \leqslant k_{2}(\rho) g_{2}(v)$ for all $v \in \mathcal{V}$, a.e. $t \in(0, T)$ and $\lim _{\rho \rightarrow 0} k_{2}(\rho)=0$,

(f) $f, f_{\rho} \in \mathcal{V}^{*}$ and $f_{\rho} \longrightarrow f$ in $\mathcal{V}^{*}$, as $\rho \longrightarrow 0$,

(g) $v_{0}, v_{0 \rho} \in V$ and $v_{0 \rho} \longrightarrow v_{0}$ in $V$, as $\rho \longrightarrow 0$.

We consider the following perturbed version of Problem 8.

Problem 12 Find $u_{\rho} \in \mathcal{W}$ such that

$$
\left\{\begin{array}{l}
u_{\rho}^{\prime}(t)+A_{\rho}\left(t, u_{\rho}(t)\right)+\left(\mathcal{S}_{\rho} u_{\rho}\right)(t)+F\left(t,\left(\mathcal{R}_{\rho} u_{\rho}\right)(t), u_{\rho}(t)\right) \ni f_{\rho}(t) \quad \text { for a.e. } t \in(0, T) \\
u_{\rho}(0)=v_{0 \rho} .
\end{array}\right.
$$

We have the following convergence result.

Theorem 13 Under assumptions (7), (8)(a), (14), $u_{0} \in V$ and

$$
m_{A}>L_{1} T+m_{F 1}+m_{F 2} L_{2} T,
$$

the solution $u_{\rho}$ of Problem 12 converges to the solution $u$ of Problem 8 in the following sense $\left\|u_{\rho}-u\right\|_{\mathcal{V}}+\left\|u_{\rho}-u\right\|_{C(0, T ; H)} \longrightarrow 0$, as $\rho \longrightarrow 0$.

Proof We observe that hypothesis (15) implies condition (8)(b). From Theorem 10 there exist $u_{\rho}, u \in \mathcal{W}$ unique solutions to Problems 12 and 8, respectively. From Definition 9, we have

$$
u_{\rho}^{\prime}(t)+A_{\rho}\left(t, u_{\rho}(t)\right)+\left(\mathcal{S}_{\rho} u_{\rho}\right)(t)+\zeta_{\rho}(t)=f_{\rho}(t)
$$




$$
u^{\prime}(t)+A(t, u(t))+(\mathcal{S} u)(t)+\zeta(t)=f(t)
$$

for a.e. $t \in(0, T)$, where $\zeta_{\rho}(t) \in F\left(t,\left(\mathcal{R}_{\rho} u_{\rho}\right)(t), u_{\rho}(t)\right)$ and $\zeta(t) \in F(t,(\mathcal{R} u)(t), u(t))$ for a.e. $t \in(0, T)$. We substract the equality (17) from (16) and multiply obtained result by $u(t)-u_{\rho}(t)$. Integrating the result over $(0, t)$, for all $t \in[0, T]$, we have

$$
\begin{aligned}
\int_{0}^{t}\left\langle u_{\rho}^{\prime}(s)-u^{\prime}(s), u(s)-u_{\rho}(s)\right\rangle_{V^{*} \times V} d s \\
\quad+\int_{0}^{t}\left\langle A_{\rho}\left(s, u_{\rho}(s)\right)-A(s, u(s)), u(s)-u_{\rho}(s)\right\rangle_{V^{*} \times V} d s \\
\quad+\int_{0}^{t}\left\langle\left(\mathcal{S}_{\rho} u_{\rho}\right)(s)-(\mathcal{S} u)(s), u(s)-u_{\rho}(s)\right\rangle_{V^{*} \times V} d s \\
\quad+\int_{0}^{t}\left\langle\zeta_{\rho}(s)-\zeta(s), u(s)-u_{\rho}(s)\right\rangle_{Z^{*} \times Z} d s \\
=\int_{0}^{t}\left\langle f_{\rho}(s)-f(s), u(s)-u_{\rho}(s)\right\rangle_{V^{*} \times V} d s .
\end{aligned}
$$

We apply the integration by parts formula, properties (1)(b) and (7)(d), and obtain

$$
\begin{aligned}
& \frac{1}{2}\left\|u_{\rho}(t)-u(t)\right\|_{H}^{2}+m_{A} \int_{0}^{t}\left\|u_{\rho}(s)-u(s)\right\|_{V}^{2} d s-m_{F 1} \int_{0}^{t}\left\|u_{\rho}(s)-u(s)\right\|_{V}^{2} d s \\
& \leqslant \int_{0}^{t}\left\|f_{\rho}(s)-f(s)\right\|_{V^{*}}\left\|u(s)-u_{\rho}(s)\right\|_{V} d s \\
& \quad+\int_{0}^{t}\left\|A_{\rho}(s, u(s))-A(s, u(s))\right\|_{V^{*}}\left\|u(s)-u_{\rho}(s)\right\|_{V} d s \\
& \quad+\int_{0}^{t}\left\|\left(\mathcal{S}_{\rho} u_{\rho}\right)(s)-\left(\mathcal{S}_{\rho} u\right)(s)\right\|_{V^{*}}\left\|u(s)-u_{\rho}(s)\right\|_{V} d s \\
& \quad+\int_{0}^{t}\left\|\left(\mathcal{S}_{\rho} u\right)(s)-(\mathcal{S} u)(s)\right\|_{V^{*}}\left\|u(s)-u_{\rho}(s)\right\|_{V} d s \\
& \quad+m_{F 2} \int_{0}^{t}\left\|\left(\mathcal{R}_{\rho} u_{\rho}\right)(s)-\left(\mathcal{R}_{\rho} u\right)(s)\right\|_{V}\left\|u(s)-u_{\rho}(s)\right\|_{V} d s \\
& \quad+m_{F 2} \int_{0}^{t}\left\|\left(\mathcal{R}_{\rho} u\right)(s)-(\mathcal{R} u)(s)\right\|_{V}\left\|u(s)-u_{\rho}(s)\right\|_{V} d s+\frac{1}{2}\left\|v_{0 \rho}-v_{0}\right\|_{H}^{2}
\end{aligned}
$$

for all $t \in[0, T]$. Omitting the first term on the left hand side, using hypothesis (14)(b), (d) and the Hölder inequality, we infer that

$$
\begin{aligned}
m_{A} \| & u_{\rho}-u\left\|_{\mathcal{V}}^{2}-m_{F 1}\right\| u_{\rho}-u \|_{\mathcal{V}}^{2} \\
\leqslant & \left\|f_{\rho}-f\right\|_{\mathcal{V}^{*}}\left\|u-u_{\rho}\right\|_{\mathcal{V}}+\left\|A_{\rho}(\cdot, u)-A(\cdot, u)\right\|_{\mathcal{V}^{*}}\left\|u-u_{\rho}\right\|_{\mathcal{V}} \\
& +L_{1} T\left\|u-u_{\rho}\right\|_{\mathcal{V}}^{2}+\left\|\mathcal{S}_{\rho} u-\mathcal{S} u\right\|_{\mathcal{V}^{*}}\left\|u-u_{\rho}\right\|_{\mathcal{V}}+m_{F 2} L_{2} T\left\|u-u_{\rho}\right\|_{\mathcal{V}}^{2} \\
& +m_{F 2}\left\|\mathcal{R}_{\rho} u-\mathcal{R} u\right\|_{\mathcal{V}}\left\|u-u_{\rho}\right\|_{\mathcal{V}}+\frac{1}{2}\left\|v_{0 \rho}-v_{0}\right\|_{H}^{2} .
\end{aligned}
$$


Hence, by hypothesis (15) and the elementary property: $x^{2} \leqslant a x+b$ implies $x^{2} \leqslant a^{2}+b$ for all $x, a, b \geqslant 0$, we get

$$
\begin{aligned}
\left\|u_{\rho}-u\right\|_{\mathcal{V}}^{2} \leqslant & c\left(\left\|f_{\rho}-f\right\|_{\mathcal{V}^{*}}+\left\|A_{\rho}(\cdot, u)-A(\cdot, u)\right\|_{\mathcal{V}^{*}}+\left\|\mathcal{S}_{\rho} u-\mathcal{S} u\right\|_{\mathcal{V}^{*}}+\left\|\mathcal{R}_{\rho} u-\mathcal{R} u\right\|_{\mathcal{V}}\right)^{2} \\
& +\left\|v_{0 \rho}-v_{0}\right\|_{H}^{2}
\end{aligned}
$$

with a constant $c$ independent of $\rho$. Since $A_{\rho}$ is a bounded operator (cf. (1)(d)), from condition (14)(a) and the Lebesgue dominated convergence theorem, we see that

$$
A_{\rho}(\cdot, u) \longrightarrow A(\cdot, u) \text { in } \mathcal{V}^{*}, \quad \text { as } \rho \longrightarrow 0 .
$$

Moreover, from conditions (14)(c), (e), we have

$$
\left\|\mathcal{S}_{\rho} u-\mathcal{S} u\right\|_{\mathcal{V}^{*}} \leqslant \sqrt{T} k_{1}(\rho) g_{1}(u) \quad \text { and } \quad\left\|\mathcal{R}_{\rho} u-\mathcal{R} u\right\|_{\mathcal{V}} \leqslant \sqrt{T} k_{2}(\rho) g_{2}(u),
$$

which imply

$$
\lim _{\rho \rightarrow 0}\left\|\mathcal{S}_{\rho} u-\mathcal{S} u\right\|_{\mathcal{V}^{*}}=0 \text { and } \quad \lim _{\rho \rightarrow 0}\left\|\mathcal{R}_{\rho} u-\mathcal{R} u\right\|_{\mathcal{V}}=0
$$

These observations, together with assumptions (14)(f) and (g), lead to the following conclusion

$$
\lim _{\rho \rightarrow 0}\left\|u_{\rho}-u\right\|_{\mathcal{V}}=0 .
$$

Finally, from (19), the Hölder inequality and assumptions (1)(b), (6), (7)(d), (15), and (14)(b), (d), we obtain

$$
\begin{aligned}
\left\|u_{\rho}(t)-u(t)\right\|_{H}^{2} \leqslant & c\left(\left\|A_{\rho}(\cdot, u)-A(\cdot, u)\right\|_{\mathcal{V}^{*}}+\left\|\mathcal{S}_{\rho} u-\mathcal{S} u\right\|_{\mathcal{V}^{*}}\right. \\
& \left.+\left\|\mathcal{R}_{\rho} u-\mathcal{R} u\right\|_{\mathcal{V}}+\left\|f_{\rho}-f\right\|_{\mathcal{V}^{*}}\right)\left\|u_{\rho}-u\right\|_{\mathcal{V}}+c\left\|v_{0 \rho}-v_{0}\right\|_{H}^{2},
\end{aligned}
$$

for all $t \in[0, T]$ with a constant $c$ independent of $\rho$. Hence and from conditions (14)(f), (g), and (20)-(22), we see that $\left\|u_{\rho}-u\right\|_{C(0, T ; H)} \longrightarrow 0$, as $\rho \longrightarrow 0$. This completes the proof of the theorem.

We note that the result of Theorem 10 on the existence and uniqueness of solution to Problem 8 holds without any restriction on the size $T$ of the time interval. On contrary, the convergence result provided in Theorem 13 requires an essential smallness condition (15) which relates the coercivity constant of the operator $A$ with the monotonicity constants $m_{F 1}$ and $m_{F 2}$, the constants for the history-dependent operators $L_{1}$ and $L_{2}$, and the length of the time interval. This condition can be violated when $T$ is sufficiently large, and therefore, the convergence result is only a local one. On the other hand, the convergence result presented in Theorem 13 represents a crucial tool in the analysis of dynamic frictional contact problems for viscoelastic materials.

\section{A Viscoelastic Contact Model}

This section is devoted to the analysis of a viscoelastic contact model with history-dependent operators. First, we deliver a result on the unique weak solvability. Then, we present a result 
concerning the dependence of the solution of Problem 17 on perturbations of the viscosity operator, the elasticity operator, the relaxation operator, the normal compliance function $p_{v}$, the memory function $b$, body forces $f_{0}$ and surface tractions $f_{2}$. In the study of this model, we use the abstract results that we obtained in Sect. 3.

We consider a body which occupies an open, bounded and connected set $\Omega \subset \mathbb{R}^{d}$, where $d=2,3$. We assume that the set $\Omega$ has a Lipschitz continuous boundary $\Gamma$. The boundary $\Gamma$ is divided into three mutually disjoint and relatively open sets $\Gamma_{1}, \Gamma_{2}$ and $\Gamma_{3}$. Moreover, we assume that meas $\left(\Gamma_{1}\right)>0$ and the body is clamped on $\Gamma_{1}$. Surface tractions of density $f_{2}$ act on the part $\Gamma_{2}$, whereas a force of density $f_{0}$ acts in $\Omega$. The body is in frictional contact on $\Gamma_{3}$ with a deformable foundation. We suppose that between the body and the foundation there is no separation. The problem is dynamic and we study the evolution process of the mechanical state of the body on the finite time interval $[0, T]$, where $T>0$.

We denote by $\mathbb{S}^{d}$ the space of second order symmetric tensors on $\mathbb{R}^{d}$. We will use the symbols $\sigma=\sigma(x, t)=\left(\sigma_{i j}(x, t)\right)$ and $u=u(x, t)=\left(u_{i}(x, t)\right)$ to denote the stress field and the displacement field, respectively. Moreover, by $v=\left(v_{i}\right)$ we denote the outward unit normal on $\Gamma$. Here, the indices $i$ and $j$ run between 1 and $d$. The functions $\sigma: \Omega \times[0, T] \longrightarrow \mathbb{S}^{d}$ and $u: \Omega \times[0, T] \longrightarrow \mathbb{R}^{d}$ are the unknowns in the contact problem. We recall that in $\mathbb{R}^{d}$ and $\mathbb{S}^{d}$, we consider the following canonical inner products and corresponding norms.

$$
\begin{aligned}
& u \cdot v=\sum_{i} u_{i} v_{i}, \quad\|v\|_{\mathbb{R}^{d}}=(v \cdot v)^{\frac{1}{2}} \quad \text { for all } u=\left(u_{i}\right), v=\left(v_{i}\right) \in \mathbb{R}^{d}, \\
& \sigma: \tau=\sum_{i j} \sigma_{i j} \tau_{i j}, \quad\|\tau\|_{\mathbb{S}^{d}}=(\tau: \tau)^{\frac{1}{2}} \quad \text { for all } \sigma=\left(\sigma_{i j}\right), \tau=\left(\tau_{i j}\right) \in \mathbb{S}^{d} .
\end{aligned}
$$

Moreover, $u^{\prime}=\frac{\partial u}{\partial t}$ and $u^{\prime \prime}=\frac{\partial^{2} u}{\partial t^{2}}$ represent the velocity field and the acceleration field, respectively. We use the standard notation for Lebesgue and Sobolev spaces. Moreover, we consider the following spaces.

$$
\begin{aligned}
& H=L^{2}\left(\Omega ; \mathbb{R}^{d}\right), \quad H_{1}=H^{1}\left(\Omega ; \mathbb{R}^{d}\right), \\
& \mathcal{H}=\left\{\tau=\left(\tau_{i j}\right) \mid \tau_{i j}=\tau_{j i} \in L^{2}(\Omega)\right\}, \quad \mathcal{H}_{1}=\left\{\tau \in \mathcal{H} \mid\left(\sum_{j} \frac{\partial \tau_{i j}}{\partial x_{j}}\right)_{i} \in H\right\}, \\
& V=\left\{v \in H_{1} \mid v=0 \text { on } \Gamma_{1}\right\}, \quad Z=H^{\delta}\left(\Omega ; \mathbb{R}^{d}\right), \text { where } \delta \in\left(\frac{1}{2}, 1\right) .
\end{aligned}
$$

Recall that $V \subset H \subset V^{*}$ and $Z \subset H \subset Z^{*}$ form the evolution triples of spaces and the embedding $V \subset Z$ is compact. We denote by $c_{\mathrm{e}}>0$ the embedding constant of $V$ into $Z$. We define the deformation operator $\varepsilon: H_{1} \longrightarrow \mathcal{H}$ by $\varepsilon(u)=\left(\varepsilon_{i j}(u)\right), \varepsilon_{i j}(u)=\frac{1}{2}\left(\frac{\partial u_{i}}{\partial x_{j}}+\frac{\partial u_{j}}{\partial x_{i}}\right)$. The spaces $H, \mathcal{H}$ and $H_{1}$ are Hilbert spaces endowed with the canonical inner products given by

$$
\begin{aligned}
\langle u, v\rangle_{H} & =\int_{\Omega} \sum_{i} u_{i} v_{i} d x=\int_{\Omega} u \cdot v d x, \quad\langle\sigma, \tau\rangle_{\mathcal{H}}=\int_{\Omega} \sum_{i j} \sigma_{i j} \tau_{i j} d x=\int_{\Omega} \sigma: \tau d x, \\
\langle u, v\rangle_{H_{1}} & =\langle u, v\rangle_{H}+\langle\varepsilon(u), \varepsilon(v)\rangle_{\mathcal{H}}
\end{aligned}
$$

and the associated norms $\|\cdot\|_{H},\|\cdot\|_{\mathcal{H}},\|\cdot\|_{H_{1}}$, respectively. The space $V$ is a real Hilbert space with the inner product given by $\langle u, v\rangle_{V}=\langle\varepsilon(u), \varepsilon(v)\rangle_{\mathcal{H}}$ for all $u, v \in V$ and the 
corresponding norm defined by $\|v\|_{V}=\|\varepsilon(v)\|_{\mathcal{H}}$ for all $v \in V$. From the assumption meas $\left(\Gamma_{1}\right)>0$, it follows that the space $V$ is complete and the Korn inequality holds, i.e., there exists $\tilde{c}>0$, which depends on $\Omega$ and $\Gamma_{1}$, such that $\|\varepsilon(v)\|_{\mathcal{H}} \geqslant \tilde{c}\|v\|_{H_{1}}$ for all $v \in V$. We denote by $\gamma: Z \longrightarrow L^{2}\left(\Gamma ; \mathbb{R}^{d}\right)$ the trace operator, by $\|\gamma\|=\|\gamma\|_{\mathcal{L}\left(Z ; L^{2}\left(\Gamma ; \mathbb{R}^{d}\right)\right)}$ its norm and by $\gamma^{*}: L^{2}\left(\Gamma ; \mathbb{R}^{d}\right) \longrightarrow Z^{*}$ the operator adjoint to $\gamma$. The normal and the tangential components of the displacement field on the boundary $\Gamma$ are defined by $v_{v}=v \cdot v$ and $v_{\tau}=v-v_{v} \cdot v$, respectively. Under the assumption that $\sigma$ is a regular function, we can define the normal and tangential components of the stress field $\sigma$ on the boundary $\Gamma$ as follows $\sigma_{v}=\sigma v \cdot v$ and $\sigma_{\tau}=\sigma v-\sigma_{v} v$. The following Green formula will be used in the derivation of variational formulation of a contact problem.

$$
\langle\sigma, \varepsilon(v)\rangle_{\mathcal{H}}+\langle\operatorname{Div} \sigma, v\rangle_{H}=\int_{\Gamma} \sigma v \cdot \gamma v d \Gamma \quad \text { for all } v \in H_{1} \text { and } \sigma \in \mathcal{H}_{1},
$$

where $\operatorname{Div} \sigma=\left(\sigma_{i j, j}\right)$ denotes the divergence operator. Moreover, the following decomposition formula holds

$$
\sigma v \cdot v=\sigma_{v} v_{v}+\sigma_{\tau} \cdot v_{\tau}
$$

To shorten the notation, we write $Q=\Omega \times(0, T)$ and $\Sigma_{i}=\Gamma_{i} \times(0, T)$ for $i=1,2,3$.

We consider the classical formulation of the contact problem.

Problem 14 Find a displacement field $u: Q \longrightarrow \mathbb{R}^{d}$ and a stress field $\sigma: Q \longrightarrow \mathbb{S}^{d}$ such that

$$
\begin{aligned}
& \sigma(t)=\mathcal{A}\left(t, \varepsilon\left(u^{\prime}(t)\right)\right)+\mathcal{B}(t, \varepsilon(u(t)))+\int_{0}^{t} \mathcal{C}(t-s) \varepsilon\left(u^{\prime}(s)\right) d s \quad \text { in } Q, \\
& u^{\prime \prime}(t)-\operatorname{Div} \sigma(t)=f_{0}(t) \quad \text { in } Q, \\
& u(t)=0 \quad \text { on } \Sigma_{1}, \\
& \sigma(t) v=f_{2}(t) \quad \text { on } \Sigma_{2}, \\
& -\sigma_{\nu}(t)=p\left(t, u_{v}(t)\right)+\int_{0}^{t} b(t-s) u_{\nu}(s) d s \quad \text { on } \Sigma_{3}, \\
& -\sigma_{\tau}(t) \in F_{b}\left(\left\|u_{\tau}(t)\right\|_{\mathbb{R}^{d}}\right) \partial j_{\tau}\left(t, u_{\tau}^{\prime}(t)\right) \quad \text { on } \Sigma_{3}, \\
& u(0)=u_{0}, \quad u^{\prime}(0)=v_{0} \quad \text { in } \Omega .
\end{aligned}
$$

We present a short description of the equations and conditions in Problem 14. Equation (25) represents the viscoelastic constitutive law in which $\mathcal{A}$ denotes the viscosity operator, $\mathcal{B}$ is the elasticity operator and $\mathcal{C}$ stands for the relaxation operator. Equation (26) is the equation of motion. Equations (27) and (28) are the displacement and the traction boundary conditions, respectively. Condition (29) was introduced in [5] and represents the contact condition. Here $p$ and $b$ are given functions which describe the instantaneous and the memory reaction of the obstacle, respectively. Condition (30) represents a friction law in which the coefficient $F_{b}=F_{b}\left(\left\|u_{\tau}(t)\right\|_{\mathbb{R}^{d}}\right)$ is a friction bound. The latter is assumed to be dependent on the magnitude of the tangential displacement. We refer to the tangential component $u_{\tau}$ as the slip, cf. [21] and [27]. The physical model of slip-dependent friction was introduced 
by Rabinowicz [24] in the geophysical context of earthquakes' modelling. This model of friction was studied in the literature by Ionescu and Paumier [12], Ionescu and Nguyen [10], Ionescu et al. [11], Shillor et al. in Chap. 10.1 of [30], Migórski [16, 17], and Migórski and Ochal [18]. The law 30 is a generalization of the Coulomb law with slip-dependent friction. In many geophysical publications the motion of tectonic plates is modeled with the Coulomb law in which the friction bound is assumed to depend on the magnitude of the tangential displacement. The friction bound $F_{b}$ depends also explicitely on $x$ which allows to describe the nonhomogeneity of the contact surface. For concrete examples of the friction law of the form (30), we refer to Sects. 6 and 7 in [21]. More detailed information about the law (30) can be found, for instance, in $[19,21,27,30]$ and the references therein. Finally, Eqs. (31) are the initial conditions, in which $u_{0}$ denotes the initial displacement and $v_{0}$ denotes the initial velocity.

In the study of Problem 14, we consider the following assumptions for operators and functions.

$\mathcal{A}: Q \times \mathbb{S}^{d} \longrightarrow \mathbb{S}^{d}$ is such that

(a) $\mathcal{A}(\cdot, \cdot, \varepsilon)$ is measurable on $Q$ for all $\varepsilon \in \mathbb{S}^{d}$,

(b) $\mathcal{A}(x, t, \cdot)$ is continuous on $\mathbb{S}^{d}$ for a.e. $(x, t) \in Q$,

(c) $\|\mathcal{A}(x, t, \varepsilon)\|_{\mathbb{S}^{d}} \leqslant \bar{a}_{0}(x, t)+\bar{a}_{1}\|\varepsilon\|_{\mathbb{S}^{d}}$ for all $\varepsilon \in \mathbb{S}^{d}$,

a.e. $(x, t) \in Q$ with $\bar{a}_{0} \in L^{2}(Q)$ and $\bar{a}_{0}, \bar{a}_{1} \geqslant 0$,

(d) $\left(\mathcal{A}\left(x, t, \varepsilon_{1}\right)-\mathcal{A}\left(x, t, \varepsilon_{2}\right)\right):\left(\varepsilon_{1}-\varepsilon_{2}\right) \geqslant m_{\mathcal{A}}\left\|\varepsilon_{1}-\varepsilon_{2}\right\|_{\mathbb{S}^{d}}^{2}$ for all $\varepsilon_{1}, \varepsilon_{2} \in \mathbb{S}^{d}$, a.e. $(x, t) \in Q$ with $m_{\mathcal{A}}>0$,

(e) $\mathcal{A}(x, t, 0)=0$ for a.e. $(x, t) \in Q$.

$\mathcal{B}: Q \times \mathbb{S}^{d} \longrightarrow \mathbb{S}^{d}$ is such that

$\left.\begin{array}{l}\text { (a) } \mathcal{B}(\cdot, \cdot, \varepsilon) \text { is measurable on } Q \text { for all } \varepsilon \in \mathbb{S}^{d} \text { and } \mathcal{B}(\cdot, \cdot, 0) \in L^{2}\left(Q ; \mathbb{S}^{d}\right) \text {, } \\ \text { (b) }\left\|\mathcal{B}\left(x, t, \varepsilon_{1}\right)-\mathcal{B}\left(x, t, \varepsilon_{2}\right)\right\|_{\mathbb{S}^{d}} \leqslant L_{\mathcal{B}}\left\|\varepsilon_{1}-\varepsilon_{2}\right\|_{\mathbb{S}^{d}} \text { for all } \varepsilon_{1}, \varepsilon_{2} \in \mathbb{S}^{d}, \\ \text { a.e. }(x, t) \in Q \text { with } L_{\mathcal{B}}>0 \text {. }\end{array}\right\}$

$\mathcal{C}: Q \times \mathbb{S}^{d} \longrightarrow \mathbb{S}^{d}$ is such that

(a) $\mathcal{C}(x, t, \varepsilon)=\left(c_{i j k l}(x, t) \varepsilon_{k l}\right)$ for all $\varepsilon=\left(\varepsilon_{i j}\right) \in \mathbb{S}^{d}$, a.e. $(x, t) \in Q$,

(b) $c_{i j k l}(x, t)=c_{j i k l}(x, t)=c_{l k i j}(x, t)$ a.e. $(x, t) \in Q$,

for all $i, j, k, l=1, \ldots, d$,

(c) $\quad c_{i j k l} \in L^{\infty}\left(0, T ; L^{\infty}(\Omega)\right)$ for all $i, j, k, l=1, \ldots, d$.

$p: \Sigma_{3} \times \mathbb{R} \longrightarrow \mathbb{R}$ is such that

(a) $p(\cdot, \cdot, r)$ is measurable on $\Sigma_{3}$ for all $r \in \mathbb{R}$ and $p(\cdot, \cdot, 0) \in L^{2}\left(\Sigma_{3}\right)$,

(b) $\left|p\left(x, t, r_{1}\right)-p\left(x, t, r_{2}\right)\right| \leqslant L_{p}\left|r_{1}-r_{2}\right|$ for all $r_{1}, r_{2} \in \mathbb{R}$, a.e. $(x, t) \in \Sigma_{3}$
with $L_{p}>0$.

$b \in L^{1}\left(0, T ; L^{\infty}\left(\Gamma_{3}\right)\right), b(x, t) \geqslant 0$ for a.e. $(x, t) \in \Sigma_{3}$. 
$F_{b}: \Gamma_{3} \times \mathbb{R} \longrightarrow \mathbb{R}$ is such that

(a) $F_{b}(\cdot, r)$ is measurable for all $r \in \mathbb{R}$,

(b) $\left|F_{b}\left(x, r_{1}\right)-F_{b}\left(x, r_{2}\right)\right| \leqslant L_{F_{b}}\left|r_{1}-r_{2}\right|$ for all $r_{1}, r_{2} \in \mathbb{R}$, a.e. $x \in \Gamma_{3}$ with $L_{F_{b}}>0$,

(c) $0 \leqslant F_{b}(x, r) \leqslant \bar{F}$ for all $r \in \mathbb{R}$, a.e. $x \in \Gamma_{3}$ with $\bar{F}>0$.

$j_{\tau}: \Sigma_{3} \times \mathbb{R}^{d} \longrightarrow \mathbb{R}$ is such that

(a) $j_{\tau}(\cdot, \cdot, \xi)$ is measurable on $\Sigma_{3}$ for all $\xi \in \mathbb{R}^{d}$, and $j_{\tau}(\cdot, \cdot, 0) \in L^{1}\left(\Sigma_{3}\right)$,

(b) $j_{\tau}(x, t, \cdot)$ is locally Lipschitz on $\mathbb{R}^{d}$ for a.e. $(x, t) \in \Sigma_{3}$,

(c) $\left\|\partial j_{\tau}(x, t, \xi)\right\|_{\mathbb{R}^{d}} \leqslant M$ for all $\xi \in \mathbb{R}^{d}$, a.e. $(x, t) \in \Sigma_{3}$ with $M>0$,

(d) $\left\|\partial j_{\tau}\left(x, t, \xi_{1}\right)-\partial j_{\tau}\left(x, t, \xi_{2}\right)\right\|_{\mathbb{R}^{d}} \leqslant L_{j_{\tau}}\left\|\xi_{1}-\xi_{2}\right\|_{\mathbb{R}^{d}}$ for all $\xi_{1}, \xi_{2} \in \mathbb{R}^{d}$, a.e. $(x, t) \in \Sigma_{3}$ with $L_{j_{\tau}}>0$,

(e) $j_{\tau}(x, t, \cdot)$ or $-j_{\tau}(x, t, \cdot)$ is regular for a.e. $(x, t) \in \Sigma_{3}$.

Below we provide concrete examples of the tangential potentials which satisfy the hypothesis (38). For simplicity, we skip the dependence on the $(x, t)$ variable.

Example 15 Let $j_{\tau}: \mathbb{R}^{d} \longrightarrow \mathbb{R}$ be defined by

$$
j_{\tau}(\xi)=\sqrt{\|\xi\|_{\mathbb{R}^{d}}^{2}+\rho^{2}}-\rho,
$$

for $\xi \in \mathbb{R}^{d}$, where $\rho>0$ is a fixed regularization parameter. The function $j_{\tau}$ is convex and Gateaux differentiable with $\partial j_{\tau}(\xi)=\frac{\xi}{\sqrt{\|\xi\|^{2}+\rho^{2}}}$ for $\xi \in \mathbb{R}^{d}$, and it satisfies hypothesis (38) with $M=1$ and $L_{j_{\tau}}=\frac{1}{\rho}$. In this case, the friction condition (30) reduces to the following law

$$
-\sigma_{\tau}(t)=F_{b}\left(\left\|u_{\tau}(t)\right\|_{\mathbb{R}^{d}}\right) \frac{u_{\tau}^{\prime}}{\sqrt{\left\|u_{\tau}^{\prime}(t)\right\|_{\mathbb{R}^{d}}^{2}+\rho^{2}}} \text { on } \Sigma_{3} .
$$

This condition represents a regularization of the Coulomb law which is used in the literature for numerical reasons, cf. [30]. For more discussion, see Chap. 6 of [21].

Example 16 Let $j_{\tau}: \mathbb{R}^{d} \longrightarrow \mathbb{R}$ be defined by

$$
j_{\tau}(\xi)= \begin{cases}\frac{1}{2}\|\xi\|_{\mathbb{R}^{d}}^{2}, & \text { if }\|\xi\|_{\mathbb{R}^{d}} \leqslant L_{0} \\ L_{0}\|\xi\|_{\mathbb{R}^{d}}-\frac{L_{0}^{2}}{2}, & \text { otherwise }\end{cases}
$$

for $\xi \in \mathbb{R}^{d}$, where $L_{0}>0$. Then

$$
\partial j_{\tau}(\xi)= \begin{cases}\xi, & \text { if }\|\xi\|_{\mathbb{R}^{d}} \leqslant L_{0} \\ L_{0} \frac{\xi}{\|\xi\|_{\mathbb{R}^{d}}}, & \text { otherwise }\end{cases}
$$

for $\xi \in \mathbb{R}^{d}$. It is clear that $j_{\tau}$ satisfies hypotheses (38) with $M=L_{0}$ and $L_{j_{\tau}}=1$. In this case, the friction condition (30) takes the following form

$$
-\sigma_{\tau}(t)=F_{b}\left(\left\|u_{\tau}(t)\right\|_{\mathbb{R}^{d}}\right) R\left(u_{\tau}^{\prime}(t)\right) \quad \text { on } \Sigma_{3},
$$


where $R: \mathbb{R}^{d} \longrightarrow \mathbb{R}^{d}$ is the truncation operator given by

$$
R(\xi)= \begin{cases}\xi, & \text { if }\|\xi\|_{\mathbb{R}^{d}} \leqslant L_{0} \\ L_{0} \frac{\xi}{\|\xi\|_{\mathbb{R}^{d}}}, & \text { if }\|\xi\|_{\mathbb{R}^{d}}>L_{0}\end{cases}
$$

for $\xi \in \mathbb{R}^{d}$. The constant $L_{0}$ is a limit bound constant, cf. [4] and [30].

Moreover, we assume that the body forces and tractions in Problem 14 have the following regularity

$$
f_{0} \in L^{2}\left(0, T ; L^{2}\left(\Omega ; \mathbb{R}^{d}\right)\right), \quad f_{2} \in L^{2}\left(0, T ; L^{2}\left(\Gamma_{2} ; \mathbb{R}^{d}\right)\right) .
$$

The initial displacement and the initial velocity are such that

$$
u_{0}, v_{0} \in V
$$

Next, we present a variational formulation of Problem 14. Let $(u, \sigma)$ be a couple of sufficiently smooth functions which satisfy Problem 14 . Let $t \in(0, T)$ and $v \in V$. Using the Green formula (23) and the equation of motion (26), we obtain

$$
\left\langle u^{\prime \prime}(t), v\right\rangle_{V^{*} \times V}+\langle\sigma(t), \varepsilon(v)\rangle_{\mathcal{H}}=\left\langle f_{0}(t), v\right\rangle_{H}+\int_{\Gamma} \sigma(t) v \cdot \gamma v d \Gamma
$$

From conditions (27), (28) and the decomposition formula (24), we get

$$
\int_{\Gamma} \sigma(t) v \cdot \gamma v d \Gamma=\left\langle f_{2}(t), \gamma v\right\rangle_{L^{2}\left(\Gamma_{2} ; \mathbb{R}^{d}\right)}+\int_{\Gamma_{3}}\left(\sigma_{v}(t) v_{v}+\sigma_{\tau}(t) \cdot v_{\tau}\right) d \Gamma
$$

Based on the definition of the Clarke subdifferential and the boundary condition (30), the following inequality holds

$$
\int_{\Gamma_{3}} \sigma_{\tau}(t) \cdot v_{\tau} d \Gamma \geqslant-\int_{\Gamma_{3}} F_{b}\left(\left\|u_{\tau}(t)\right\|_{\mathbb{R}^{d}}\right) j_{\tau}^{0}\left(t, u_{\tau}^{\prime}(t) ; v_{\tau}\right) d \Gamma
$$

On the other hand, from the boundary condition (29), we have

$$
\int_{\Gamma_{3}} \sigma_{\nu}(t) v_{\nu} d \Gamma=\int_{\Gamma_{3}} p\left(t, u_{\nu}(t)\right) v_{\nu} d \Gamma+\int_{\Gamma_{3}}\left(\int_{0}^{t} b(t-s) u_{v}(s) d s\right) v_{\nu} d \Gamma .
$$

Let $f:(0, T) \longrightarrow V^{*}$ be given by

$$
\langle f(t), v\rangle_{V^{*} \times V}=\left\langle f_{0}(t), v\right\rangle_{H}+\left\langle f_{2}(t), \gamma v\right\rangle_{L^{2}\left(\Gamma_{2} ; \mathbb{R}^{d}\right)}
$$

for all $v \in V$ and a.e. $t \in(0, T)$. Combining (41)-(45) and (25), we obtain the following variational formulation of Problem 14.

Problem 17 Find a displacement field $u \in \mathcal{V}$ such that $u^{\prime} \in \mathcal{W}$ and

$$
\begin{aligned}
& \left\langle u^{\prime \prime}(t), v\right\rangle_{V^{*} \times V}+\left\langle\mathcal{A}\left(t, \varepsilon\left(u^{\prime}(t)\right)\right), \varepsilon(v)\right\rangle_{\mathcal{H}}+\langle\mathcal{B}(t, \varepsilon(u(t))), \varepsilon(v)\rangle_{\mathcal{H}} \\
& +\left\langle\int_{0}^{t} \mathcal{C}(t-s) \varepsilon\left(u^{\prime}(s)\right) d s, \varepsilon(v)\right\rangle_{\mathcal{H}}
\end{aligned}
$$




$$
\begin{aligned}
& +\int_{\Gamma_{3}} p\left(t, u_{v}(t)\right) v_{v} d \Gamma+\int_{\Gamma_{3}}\left(\int_{0}^{t} b(t-s) u_{v}(s) d s\right) v_{\nu} d \Gamma \\
& +\int_{\Gamma_{3}} F_{b}\left(\left\|u_{\tau}(t)\right\|_{\mathbb{R}^{d}}\right) j_{\tau}^{0}\left(t, u_{\tau}^{\prime}(t) ; v_{\tau}\right) d \Gamma \geqslant\langle f(t), v\rangle_{V^{*} \times V}
\end{aligned}
$$

for all $v \in V$ and a.e. $t \in(0, T)$ with $u(0)=u_{0}$ and $u^{\prime}(0)=v_{0}$.

Problem 17 has a general form of an evolutionary hemivariational inequality of second order. Such inequalities were used to model various contact problems and studied in several papers, cf. [21] and the references therein. We note that results on the unique solvability of Problem 17 are not available in the literature. The main difficulty lies in the nonlinear term involving the product of the functions $F_{b}$ and $j_{\tau}^{0}$. All results on the unique solvability of several classes of hemivariational inequalities presented, e.g., in [21], assume that $F_{b}$ is a constant and $\partial j_{\tau}$ satisfies the relaxed monotonicity condition and has a sublinear growth. In comparison to other contributions, in the present paper, we reject the hypothesis that $F_{b}$ is a constant and assume that the friction bound $F_{b}$ depends on the slip. For this reason, we need to consider a more restrictive assumption than in [21], on the potential $j_{\tau}$, cf. (38). The unique solvability of Problem 17 without the hypotheses (38)(c) and (d) represents an interesting open problem.

Our main result on the unique solvability of Problem 17 reads as follows.

Theorem 18 Under assumptions (32)-(40) and the following smallness condition

$$
m_{\mathcal{A}}>\bar{F} L_{j_{\tau}} c_{\mathrm{e}}^{2}\|\gamma\|^{2}
$$

Problem 17 has a unique solution.

Proof The proof is based on Theorem 10. To use it, we first rewrite Problem 17 in an equivalent form of a first order hemivariational inequality for the velocity field. Let $w=u^{\prime}$. Then, by using the initial condition (40), we have

$$
u(t)=\int_{0}^{t} w(s) d s+u_{0} \quad \text { for all } t \in[0, T] .
$$

We introduce the operators $\mathcal{R}, \mathcal{R}_{1}: \mathcal{V} \longrightarrow \mathcal{V}$ defined by

$$
\left(\mathcal{R}_{1} v\right)(t)=\int_{0}^{t} v(s) d s+u_{0}, \quad(\mathcal{R} v)(t)=\left(\mathcal{R}_{1} v\right)_{\tau}(t)
$$

for $v \in \mathcal{V}$ and a.e. $t \in(0, T)$. With this notation, Problem 17 can be formulated as follows.

Problem 19 Find a velocity field $w \in \mathcal{W}$ such that

$$
\begin{aligned}
& \left\langle w^{\prime}(t), v\right\rangle_{V^{*} \times V}+\langle\mathcal{A}(t, \varepsilon(w(t))), \varepsilon(v)\rangle_{\mathcal{H}}+\left\langle\mathcal{B}\left(t, \varepsilon\left(\left(\mathcal{R}_{1} w\right)(t)\right)\right), \varepsilon(v)\right\rangle_{\mathcal{H}} \\
& +\left\langle\int_{0}^{t} \mathcal{C}(t-s) \varepsilon(w(s)) d s, \varepsilon(v)\right\rangle_{\mathcal{H}}+\int_{\Gamma_{3}} p\left(t,\left(\mathcal{R}_{1} w\right)_{v}(t)\right) v_{\nu} d \Gamma \\
& +\int_{\Gamma_{3}}\left(\int_{0}^{t} b(t-s)\left(\mathcal{R}_{1} w\right)_{v}(s) d s\right) v_{\nu} d \Gamma \\
& +\int_{\Gamma_{3}} F_{b}\left(\left\|\left(\mathcal{R}_{1} w\right)_{\tau}(t)\right\|_{\mathbb{R}^{d}}\right) j_{\tau}^{0}\left(t, w_{\tau}(t) ; v_{\tau}\right) d \Gamma \geqslant\langle f(t), v\rangle_{V^{*} \times V}
\end{aligned}
$$

for all $v \in V$ and a.e. $t \in(0, T)$ with $w(0)=v_{0}$. 
It is clear that $w \in \mathcal{W}$ satisfies (50) if and only if $u \in \mathcal{V}$ such that $u^{\prime} \in \mathcal{W}$ satisfies (46).

Next, we associate with Problem 19, an abstract evolutionary inclusion of the form as in Problem 8. To this end, we introduce the notation for this abstract inclusion. Let $A:(0, T) \times$ $V \longrightarrow V^{*}$ be defined by

$$
\langle A(t, u), v\rangle_{V^{*} \times V}=\langle\mathcal{A}(t, \varepsilon(u)), \varepsilon(v)\rangle_{\mathcal{H}} \quad \text { for } v, u \in V \text { and a.e. } t \in(0, T) .
$$

Exploiting Theorem 10 in [19], we infer, that under hypothesis (32), the operator $A$ satisfies (1) with $m_{\mathcal{A}}=m_{A}, a_{0}(t)=\sqrt{2}\left\|\bar{a}_{0}(t)\right\|_{L^{2}(\Omega)}$ and $a_{1}=\bar{a}_{1} \sqrt{2}$. Next, from the following inequality

$$
\begin{aligned}
& \left\|\left(\mathcal{R} u_{1}\right)(t)-\left(\mathcal{R} u_{2}\right)(t)\right\|_{V} \\
& \quad=\left\|\left(\mathcal{R}_{1} u_{1}\right)_{\tau}(t)-\left(\mathcal{R}_{1} u_{2}\right)_{\tau}(t)\right\|_{L^{2}\left(\Gamma_{3}\right)} \\
& \quad \leqslant\left\|\gamma\left(\left(\mathcal{R}_{1} u_{1}\right)(t)-\left(\mathcal{R}_{1} u_{2}\right)(t)\right)\right\|_{L^{2}\left(\Gamma_{3} ; \mathbb{R}^{d}\right)} \leqslant c_{\mathrm{e}}\|\gamma\|\left\|\left(\mathcal{R}_{1} u_{1}\right)(t)-\left(\mathcal{R}_{1} u_{2}\right)(t)\right\|_{V} \\
& \quad \leqslant c_{\mathrm{e}}\|\gamma\| \int_{0}^{t}\left\|u_{1}(s)-u_{2}(s)\right\|_{V} d s \quad \text { for } u_{1}, u_{2} \in \mathcal{V}, \text { a.e. } t \in(0, T),
\end{aligned}
$$

we deduce that the operator $\mathcal{R}$ satisfies (5) with $L_{\mathcal{R}}=c_{\mathrm{e}}\|\gamma\|$. Subsequently, let $\mathcal{S}: \mathcal{V} \longrightarrow \mathcal{V}^{*}$ be the operator given by

$$
\begin{aligned}
\langle(\mathcal{S} u)(t), v\rangle_{V^{*} \times V}= & \left\langle\mathcal{B}\left(t, \varepsilon\left(\left(\mathcal{R}_{1} u\right)(t)\right)\right), \varepsilon(v)\right\rangle_{\mathcal{H}}+\left\langle\int_{0}^{t} \mathcal{C}(t-s) \varepsilon(u(s)) d s, \varepsilon(v)\right\rangle_{\mathcal{H}} \\
& +\int_{\Gamma_{3}} p\left(t,\left(\mathcal{R}_{1} u\right)_{v}(t)\right) v_{\nu} d \Gamma \\
& +\int_{\Gamma_{3}}\left(\int_{0}^{t} b(t-s)\left(\mathcal{R}_{1} u\right)_{v}(s) d s\right) v_{\nu} d \Gamma
\end{aligned}
$$

for $u \in \mathcal{V}, v \in V$ and a.e. $t \in(0, T)$. From hypotheses (33)-(36) and (52), we obtain the following inequalities

$$
\begin{aligned}
& \left\langle\mathcal{B}\left(t, \varepsilon\left(\left(\mathcal{R}_{1} u_{1}\right)(t)\right)\right)-\mathcal{B}\left(t, \varepsilon\left(\left(\mathcal{R}_{1} u_{2}\right)(t)\right)\right), \varepsilon(v)\right\rangle_{\mathcal{H}} \leqslant L_{\mathcal{B}}\left(\int_{0}^{t}\left\|u_{1}(s)-u_{2}(s)\right\|_{V} d s\right)\|v\|_{V} \\
& \left\langle\int_{0}^{t} \mathcal{C}(t-s) \varepsilon\left(u_{1}(s)\right)-\varepsilon\left(u_{2}(s)\right) d s, \varepsilon(v)\right\rangle_{\mathcal{H}} \\
& \quad \leqslant\|\mathcal{C}\|_{L^{\infty}\left(Q ; \mathbb{S}^{d}\right)}\left(\int_{0}^{t}\left\|u_{1}(s)-u_{2}(s)\right\|_{V} d s\right)\|v\|_{V} \\
& \int_{\Gamma_{3}}\left(p\left(t,\left(\mathcal{R}_{1} u_{1}\right)_{v}(t)\right)-p\left(t,\left(\mathcal{R}_{1} u_{2}\right)_{v}(t)\right)\right) v_{v} d \Gamma \\
& \quad \leqslant L_{p} c_{\mathrm{e}}^{2}\|\gamma\|^{2}\left(\int_{0}^{t}\left\|u_{1}(s)-u_{2}(s)\right\|_{V} d s\right)\|v\|_{V} \\
& \int_{\Gamma_{3}}\left(\int_{0}^{t} b(t-s)\left(\left(\mathcal{R}_{1} u_{1}\right)_{v}(s)-\left(\mathcal{R}_{1} u_{2}\right)_{v}(s)\right) d s\right) v_{\nu} d \Gamma \\
& \leqslant\|b\|_{L^{1}\left(0, T ; L^{\infty}\left(\Gamma_{3}\right)\right)} c_{\mathrm{e}}^{2}\|\gamma\|^{2}\left(\int_{0}^{t}\left\|u_{1}(s)-u_{2}(s)\right\|_{V} d s\right)\|v\|_{V}
\end{aligned}
$$


for $u_{1}, u_{2} \in \mathcal{V}, v \in V$, a.e. $t \in(0, T)$. Hence, we infer that the operator $\mathcal{S}$ is well defined, and since the sum of history-dependent operators is a history-dependent operator, it satisfies (6) with $L_{\mathcal{S}}=L_{\mathcal{B}}+\|\mathcal{C}\|_{L^{\infty}\left(Q ; \mathbb{S}^{d}\right)}+\left(L_{p}+\|b\|_{L^{1}\left(0, T ; L^{\infty}\left(\Gamma_{3}\right)\right)}\right) c_{\mathrm{e}}^{2}\|\gamma\|^{2}$ (for more details, cf. also [5]).

Next, we introduce the functional $J:(0, T) \times L^{2}\left(\Gamma_{3} ; \mathbb{R}^{d}\right)^{2} \longrightarrow \mathbb{R}$ defined by

$$
J(t, w, v)=\int_{\Gamma_{3}} F_{b}\left(x,\|w(x)\|_{\mathbb{R}^{d}}\right) j_{\tau}\left(x, t, v_{\tau}(x)\right) d \Gamma
$$

for all $w, v \in L^{2}\left(\Gamma_{3} ; \mathbb{R}^{d}\right)$, a.e. $t \in(0, T)$. Let $j: \Sigma_{3} \times \mathbb{R} \times \mathbb{R}^{d} \longrightarrow \mathbb{R}$ be given by $j(x, t, r, \xi)=F_{b}(x, r) j_{\tau}(x, t, \xi)$ for $r \in \mathbb{R}, \xi \in \mathbb{R}^{d}$, a.e. $(x, t) \in \Sigma_{3}$. From hypotheses (37) and (38), the function $j$ has the following properties.

(a) $j(\cdot, \cdot, r, \xi)$ is measurable on $\Sigma_{3}$ for all $(r, \xi) \in \mathbb{R} \times \mathbb{R}^{d}$, $j_{\tau}(\cdot, \cdot, 0,0) \in L^{1}\left(\Sigma_{3}\right)$,

(b) $j(x, t, \cdot, \xi)$ is continuous for all $\xi \in \mathbb{R}^{d}$, a.e. $(x, t) \in \Sigma_{3}$,

(c) $j(x, t, r, \cdot)$ is locally Lipschitz on $\mathbb{R}^{d}$ for all $r \in \mathbb{R}$, a.e. $(x, t) \in \Sigma_{3}$,

(d) $\|\partial j(x, t, r, \xi)\|_{\mathbb{R}^{d}} \leqslant \bar{F} M$ for all $(r, \xi) \in \mathbb{R} \times \mathbb{R}^{d}$, a.e. $(x, t) \in \Sigma_{3}$,

(e) either $j(x, t, r, \cdot)$ or $-j(x, t, r, \cdot)$ is regular for all $r \in \mathbb{R}$, a.e. $(x, t) \in \Sigma_{3}$,

(f) $j^{0}(x, t, \cdot, \cdot ; \eta)$ is upper semicontinuous on $\mathbb{R} \times \mathbb{R}^{d}$, for all $\eta \in \mathbb{R}^{d}$, a.e. $(x, t) \in \Sigma_{3}$,

(g) $\quad\left(\bar{\xi}_{1}-\bar{\xi}_{2}\right) \cdot\left(\xi_{1}-\xi_{2}\right) \geqslant-\bar{F} L_{j_{\tau}}\left\|\xi_{1}-\xi_{2}\right\|_{\mathbb{R}^{d}}^{2}-L_{F_{b}} M\left\|\xi_{1}-\xi_{2}\right\|_{\mathbb{R}^{d}}\left|r_{1}-r_{2}\right|$ for all $\bar{\xi}_{i} \in \partial j\left(x, t, r_{i}, \xi_{i}\right),\left(r_{i}, \xi_{i}\right) \in \mathbb{R} \times \mathbb{R}^{d}, i=1,2$, a.e. $(x, t) \in \Sigma_{3}$.

The properties (a)-(e) are obvious consequences of the hypotheses. We show the condition (f). Let $\left(r_{n}, \xi_{n}\right),(r, \xi) \in \mathbb{R} \times \mathbb{R}^{d},\left(r_{n}, \xi_{n}\right) \longrightarrow(r, \xi)$ in $\mathbb{R} \times \mathbb{R}^{d}$ and $\eta \in \mathbb{R}^{d}$. We have

$$
\begin{aligned}
\limsup j^{0}\left(x, t, r_{n}, \xi_{n} ; \eta\right)= & \limsup F_{b}\left(x, r_{n}\right) j_{\tau}^{0}\left(x, t, \xi_{n} ; \eta\right) \\
\leqslant & \limsup \left(F_{b}\left(x, r_{n}\right)-F_{b}(x, r)\right) j_{\tau}^{0}\left(x, t, \xi_{n} ; \eta\right) \\
& +\limsup F_{b}(x, r) j_{\tau}^{0}\left(x, t, \xi_{n} ; \eta\right) \\
\leqslant & \limsup \left|F_{b}\left(x, r_{n}\right)-F_{b}(x, r)\right|\left\|\partial j\left(x, t, \xi_{n}\right)\right\|_{\mathbb{R}^{d}}\|\eta\|_{\mathbb{R}^{d}} \\
& +F_{b}(x, r) \limsup j_{\tau}^{0}\left(x, t, \xi_{n} ; \eta\right) \\
\leqslant & F_{b}(x, r) j_{\tau}^{0}(x, t, \xi ; \eta)=j^{0}(x, t, r, \xi ; \eta)
\end{aligned}
$$

for a.e. $(x, t) \in \Sigma_{3}$. In the last inequality, we have used the fact that $j_{\tau}^{0}(x, t, \cdot ; \eta)$ is upper semicontinuous for all $\eta \in \mathbb{R}$, a.e. $(x, t) \in \Sigma_{3}$, cf. Proposition 3.23 (ii) of [21]. This proves the property (f).

Finally, the property $(\mathrm{g})$ follows immediately from the following inequality

$$
\begin{aligned}
& \left|\left(\bar{\xi}_{1}-\bar{\xi}_{2}\right) \cdot\left(\xi_{1}-\xi_{2}\right)\right| \leqslant\left\|F_{b}\left(x, r_{1}\right) \partial j_{\tau}\left(x, t, \xi_{1}\right)-F_{b}\left(x, r_{2}\right) \partial j_{\tau}\left(x, t, \xi_{2}\right)\right\|_{\mathbb{R}^{d}}\left\|\xi_{1}-\xi_{2}\right\|_{\mathbb{R}^{d}} \\
& \quad \leqslant\left(\left|F_{b}\left(x, r_{1}\right)-F_{b}\left(x, r_{2}\right)\right|\left\|\partial j_{\tau}\left(x, t, \xi_{1}\right)\right\|_{\mathbb{R}^{d}}\right.
\end{aligned}
$$




$$
\begin{aligned}
& \left.+\left|F_{b}\left(x, r_{2}\right)\right|\left\|\partial j_{\tau}\left(x, t, \xi_{1}\right)-\partial j_{\tau}\left(x, t, \xi_{2}\right)\right\|_{\mathbb{R}^{d}}\right)\left\|\xi_{1}-\xi_{2}\right\|_{\mathbb{R}^{d}} \\
\leqslant & \left(M L_{F_{b}}\left|r_{1}-r_{2}\right|+\bar{F} L_{j_{\tau}}\left\|\xi_{1}-\xi_{2}\right\|_{\mathbb{R}^{d}}\right)\left\|\xi_{1}-\xi_{2}\right\|_{\mathbb{R}^{d}},
\end{aligned}
$$

for all $\bar{\xi}_{i} \in \partial j\left(x, t, r_{i}, \xi_{i}\right),\left(r_{i}, \xi_{i}\right) \in \mathbb{R} \times \mathbb{R}^{d}, i=1,2$.

From the properties (55)(a)-(f) of the function $j$, by applying Lemma 10 in [16], we obtain that the functional $J(t, w, v)=\int_{\Gamma_{3}} j\left(x, t,\|w(x)\|_{\mathbb{R}^{d}}, v_{\tau}(x)\right) d \Gamma$ for $w, v, \in L^{2}\left(\Gamma_{3} ; \mathbb{R}^{d}\right)$, a.e. $t \in(0, T)$, has the following properties.

(a) $J(\cdot, w, v)$ is measurable on $(0, T)$ for all $w, v \in L^{2}\left(\Gamma_{3} ; \mathbb{R}^{d}\right)$,

(b) $J(t, w, \cdot)$ is locally Lipschitz on $L^{2}\left(\Gamma_{3} ; \mathbb{R}^{d}\right)$

(in fact, Lipschitz on bounded subsets of $L^{2}\left(\Gamma_{3} ; \mathbb{R}^{d}\right)$ )

for all $w \in L^{2}\left(\Gamma_{3} ; \mathbb{R}^{d}\right)$, a.e. $t \in(0, T)$,

(c) $\|\partial J(t, w, v)\|_{\left(L^{2}\left(\Gamma_{3} ; \mathbb{R}^{d}\right)\right)^{*}} \leqslant \bar{F} M \operatorname{meas}\left(\Gamma_{3}\right)$ for all $w, v \in L^{2}\left(\Gamma_{3} ; \mathbb{R}^{d}\right)$,

a.e. $t \in(0, T)$,

(d) $\quad J^{0}(t, w, v ; z)=\int_{\Gamma_{3}} F_{b}\left(x,\|w(x)\|_{\mathbb{R}^{d}}\right) j_{\tau}^{0}\left(x, t, v_{\tau} ; z_{\tau}\right) d \Gamma$ for all $w, v, z \in L^{2}\left(\Gamma_{3} ; \mathbb{R}^{d}\right)$, a.e. $t \in(0, T)$,

(e) $\partial J(t, \cdot, \cdot)$ has a closed graph in $L^{2}\left(\Gamma_{3} ; \mathbb{R}^{d}\right)^{2} \times\left(w-L^{2}\left(\Gamma_{3} ; \mathbb{R}^{d}\right)\right)$ topology, for a.e. $t \in(0, T)$,

(f) either $J(t, w, \cdot)$ or $-J(t, w, \cdot)$ is regular on $L^{2}\left(\Gamma_{3} ; \mathbb{R}^{d}\right)$ for all $w \in L^{2}\left(\Gamma_{3} ; \mathbb{R}^{d}\right)$, a.e. $t \in(0, T)$.

Moreover, the functional $J$ satisfies the following condition.

$$
\begin{aligned}
\left\langle\eta_{1}-\eta_{2}, v_{1}-v_{2}\right\rangle_{L^{2}\left(\Gamma_{3} ; \mathbb{R}^{d}\right)} \geqslant & -c_{1 J}\left\|v_{1}-v_{2}\right\|_{L^{2}\left(\Gamma_{3} ; \mathbb{R}^{d}\right)}^{2} \\
& -c_{2 J}\left\|v_{1}-v_{2}\right\|_{L^{2}\left(\Gamma_{3} ; \mathbb{R}^{d}\right)}\left\|w_{1}-w_{2}\right\|_{L^{2}\left(\Gamma_{3} ; \mathbb{R}^{d}\right)}
\end{aligned}
$$

for all $\eta_{i} \in \partial J\left(t, w_{i}, v_{i}\right), w_{i}, v_{i}, \eta_{i} \in L^{2}\left(\Gamma_{3} ; \mathbb{R}^{d}\right), i=1,2$, a.e. $t \in(0, T)$ with $c_{1 J}=$ $\bar{F} L_{j_{\tau}}$ and $c_{2 J}=M L_{F_{b}}$. In order to show the condition (57), let $w_{i}, v_{i}, \eta_{i} \in L^{2}\left(\Gamma_{3} ; \mathbb{R}^{d}\right)$, $\eta_{i} \in \partial J\left(t, w_{i}, v_{i}\right), i=1$, 2. From Theorem 3.47 in [21], we know that there exists $\zeta_{i} \in$ $L^{2}\left(\Gamma_{3} ; \mathbb{R}^{d}\right)$ such that $\zeta_{i}(x) \in\left[\partial j\left(x, t,\left\|w_{i}(x)\right\|, v_{i \tau}(x)\right)\right]_{\tau}, i=1,2$, for a.e. $(x, t) \in \Sigma_{3}$ and

$$
\left\langle\eta_{i}, z\right\rangle_{L^{2}\left(\Gamma_{3} ; \mathbb{R}^{d}\right)}=\int_{\Gamma_{3}} \zeta_{i}(x) \cdot z(x) d \Gamma \quad \text { for all } z \in L^{2}\left(\Gamma_{3} ; \mathbb{R}^{d}\right) .
$$

Then, we have $\zeta_{i}(x)=z_{i \tau}(x)$ with $z_{i}(x) \in \partial j\left(x, t,\left\|w_{i}(x)\right\|, v_{i \tau}(x)\right), i=1$, 2, for a.e. $(x, t) \in \Sigma_{3}$, and from the property $(55)(\mathrm{g})$ of the function $j$ and the Hölder inequality, we obtain

$$
\begin{aligned}
\left\langle\eta_{1}\right. & \left.-\eta_{2}, v_{1}-v_{2}\right\rangle_{L^{2}\left(\Gamma_{3} ; \mathbb{R}^{d}\right)} \\
& =\int_{\Gamma_{3}}\left(\zeta_{1}(x)-\zeta_{2}(x)\right) \cdot\left(v_{1}(x)-v_{2}(x)\right) d \Gamma \\
& =\int_{\Gamma_{3}}\left(z_{1 \tau}(x)-z_{2 \tau}(x)\right) \cdot\left(v_{1}(x)-v_{2}(x)\right) d \Gamma \\
& =\int_{\Gamma_{3}}\left(z_{1}(x)-z_{2}(x)\right) \cdot\left(v_{1 \tau}(x)-v_{2 \tau}(x)\right) d \Gamma
\end{aligned}
$$




$$
\begin{aligned}
\geqslant & -\bar{F} L_{j_{\tau}} \int_{\Gamma_{3}}\left\|v_{1 \tau}(x)-v_{2 \tau}(x)\right\|_{\mathbb{R}^{d}}^{2} d \Gamma \\
& -M L_{F_{b}} \int_{\Gamma_{3}}\left\|v_{1 \tau}(x)-v_{2 \tau}(x)\right\|_{\mathbb{R}^{d}}\left|\left\|w_{1}(x)\right\|_{\mathbb{R}^{d}}-\left\|w_{2}(x)\right\|_{\mathbb{R}^{d}}\right| d \Gamma \\
\geqslant & -\bar{F} L_{j_{\tau}}\left\|v_{1}-v_{2}\right\|_{L^{2}\left(\Gamma_{3} ; \mathbb{R}^{d}\right)}^{2}-M L_{F_{b}}\left\|v_{1}-v_{2}\right\|_{L^{2}\left(\Gamma_{3} ; \mathbb{R}^{d}\right)}\left\|w_{1}-w_{2}\right\|_{L^{2}\left(\Gamma_{3} ; \mathbb{R}^{d}\right)}
\end{aligned}
$$

for a.e. $t \in(0, T)$. Hence $J$ satisfies the condition (57).

Subsequently, we define the multifunction $F:(0, T) \times V \times V \longrightarrow 2^{Z^{*}}$ by

$$
F(t, u, v)=\gamma^{*} \partial J(t, \gamma u, \gamma v) \quad \text { for } u, v, \in V \text {, a.e. } t \in(0, T),
$$

where, recall, $\gamma: Z \longrightarrow L^{2}\left(\Gamma_{3} ; \mathbb{R}^{d}\right)$ denotes the trace operator. From the properties (56) and (57) of the functional $J$ and the properties of the Clarke generalized gradient, we infer the following properties of the multifunction $F$.

(I) the values of $F$ are nonempty, closed and convex sets in $Z^{*}$,

(II) $F(\cdot, u, v)$ is measurable for all $u, v \in V$; cf. Proposition 3.44 of [21],

(III) $F(t, \cdot, \cdot)$ is upper semicontinuous from $V \times V$ to $\left(w-Z^{*}\right)$ topology, for a.e. $t \in(0, T)$; this follows from property (56)(e) of the functional $J$ and Theorem 5.13 of [21],

(IV) $\|F(t, u, v)\|_{Z^{*}} \leqslant\left\|\gamma^{*}\right\| \bar{F} M$ meas $\left(\Gamma_{3}\right)$ for all $u, v \in V$, a.e. $t \in(0, T)$; this follows from property (56)(c) of the functional $J$ and the continuity of the trace operator, (V)

$$
\begin{aligned}
& \left\langle F\left(t, u_{1}, v_{1}\right)-F\left(t, u_{2}, v_{2}\right), v_{1}-v_{2}\right\rangle_{Z^{*} \times Z} \\
& \quad \geqslant\left\langle\gamma^{*} \eta_{1}-\gamma^{*} \eta_{2}, v_{1}-v_{2}\right\rangle_{Z^{*} \times Z}=\left\langle\eta_{1}-\eta_{2}, \gamma v_{1}-\gamma v_{2}\right\rangle_{L^{2}\left(\Gamma_{3} ; \mathbb{R}^{d}\right)} \\
& \geqslant-c_{1 J}\left\|\gamma v_{1}-\gamma v_{2}\right\|_{L^{2}\left(\Gamma_{3} ; \mathbb{R}^{d}\right)}^{2}-c_{2 J}\left\|\gamma v_{1}-\gamma v_{2}\right\|_{L^{2}\left(\Gamma_{3} ; \mathbb{R}^{d}\right)}\left\|\gamma u_{1}-\gamma u_{2}\right\|_{L^{2}\left(\Gamma_{3} ; \mathbb{R}^{d}\right)} \\
& \geqslant-c_{1 J} c_{\mathrm{e}}^{2}\|\gamma\|^{2}\left\|v_{1}-v_{2}\right\|_{V}^{2}-c_{2 J} c_{\mathrm{e}}^{2}\|\gamma\|^{2}\left\|v_{1}-v_{2}\right\|_{V}\left\|u_{1}-u_{2}\right\|_{V},
\end{aligned}
$$

where $\eta_{i} \in \partial J\left(t, \gamma u_{i}, \gamma v_{i}\right), \eta_{i} \in L^{2}\left(\Gamma_{3} ; \mathbb{R}^{d}\right)$ for all $u_{i}, v_{i} \in V, i=1$, 2, a.e. $t \in(0, T)$. Here, we have used the property (57) of $J$. Hence, we deduce that the multifunction $F$ satisfies the hypothesis (7) with $d_{0}(t)=\left\|\gamma^{*}\right\| \bar{F} M$ meas $\left(\Gamma_{3}\right), d_{1}=d_{2}=0, m_{F 1}=\bar{F} L_{j_{\tau}} c_{\mathrm{e}}^{2}\|\gamma\|^{2}$ and $m_{F 2}=M L_{F_{b}} c_{\mathrm{e}}^{2}\|\gamma\|^{2}$.

Next, we consider the following inclusion associated with Problem 19.

$$
\left\{\begin{array}{l}
\text { Find } w \in \mathcal{W} \text { such that } \\
w^{\prime}(t)+A(t, w(t))+(\mathcal{S} w)(t)+F(t,(\mathcal{R} w)(t), w(t)) \ni f(t) \quad \text { for a.e. } t \in(0, T) \\
w(0)=v_{0}
\end{array}\right.
$$

From the definitions of $F$ and $J$, we obtain that $w \in \mathcal{W}$ is a solution to (58) if and only if $w \in$ $\mathcal{W}$ solves Problem 19. Indeed, let $w \in \mathcal{W}$ be a solution to Problem 19. From the definition of the operator $\mathcal{S}$ and the property (56)(d), it is clear that $w \in \mathcal{W}$ solves the following inequality

$$
\left\langle w^{\prime}(t)+A(t, w(t))+(\mathcal{S} w)(t), v\right\rangle_{V^{*} \times V}+J^{0}(t,(\mathcal{R} w)(t), \gamma w(t) ; \gamma v) \geqslant\langle f(t), v\rangle_{V^{*} \times V}
$$

for all $v \in V$, a.e. $t \in(0, T)$. Using Proposition 4 (i), this inequality is equivalent to

$$
\left\langle f(t)-w^{\prime}(t)-A(t, w(t))-(\mathcal{S} w)(t), v\right\rangle_{V^{*} \times V} \leqslant(J \circ \gamma)^{0}(t,(\mathcal{R} w)(t), w(t) ; v)
$$


for all $v \in V$, a.e. $t \in(0, T)$. This means, by the definition of the subdifferential, that

$$
f(t)-w^{\prime}(t)-A(t, w(t))-(\mathcal{S} w)(t) \in \partial(J \circ \gamma)(t,(\mathcal{R} w)(t), w(t))
$$

for a.e. $t \in(0, T)$. Exploiting Proposition 4 (ii) and the definition of $F$, we know that

$$
\partial(J \circ \gamma)(t,(\mathcal{R} w)(t), w(t))=\gamma^{*} \partial J(t,(\mathcal{R} w)(t), w(t))=F(t,(\mathcal{R} w)(t), w(t))
$$

for a.e. $t \in(0, T)$. Hence, we deduce that $f(t)-w^{\prime}(t)-A(t, w(t))-(\mathcal{S} w)(t) \in$ $F(t,(\mathcal{R} w)(t), w(t))$ for a.e. $t \in(0, T)$. This proves that $w \in \mathcal{W}$ is a solution to Problem 19 if and only if $u \in \mathcal{W}$ is a solution to (58).

Therefore, it is enough to establish the existence of a solution to (58). Since the operators $A, \mathcal{R}, \mathcal{S}$ and the multifunction $F$ satisfy (1), (5), (6) and (7), respectively, and the smallness condition (47) implies (3) (recall that $d_{2}=0$ ), we are in a position to apply Theorem 10 to Problem 58. We conclude that Problem 58 has a unique solution $w \in \mathcal{W}$. Finally, we obtain that there is a unique solution $u \in \mathcal{V}$ with $u^{\prime} \in \mathcal{W}$ defined by (48) to Problem 17. This completes the proof of the theorem.

Next, we study the behaviour of the solution of Problem 17 with respect to perturbations of the data. For every $\rho>0$, let $\mathcal{A}_{\rho}, \mathcal{B}_{\rho}, \mathcal{C}_{\rho}, p_{\rho}, b_{\rho}$ and $f_{\rho}$ be perturbations of $\mathcal{A}, \mathcal{B}, \mathcal{C}, p, b$ and $f$, respectively. We need the following hypotheses.

(a) the operator $\mathcal{A}_{\rho}$ satisfies (32) and $\mathcal{A}_{\rho}(x, t, \varepsilon) \longrightarrow \mathcal{A}(x, t, \varepsilon)$ in $\mathbb{S}^{d}$ for all $\varepsilon \in \mathbb{S}^{d}$, a.e. $(x, t) \in Q$, as $\rho \longrightarrow 0$

(b) the operator $\mathcal{C}_{\rho}$ satisfies (34) and $\lim _{\rho \rightarrow 0}\left\|\mathcal{C}_{\rho}-\mathcal{C}\right\|_{L^{\infty}\left(Q ; \mathbb{S}^{d}\right)}=0$,

(c) the function $b_{\rho}$ satisfies (36) and $\lim _{\rho \rightarrow 0}\left\|b_{\rho}-b\right\|_{L^{1}\left(0, T ; L^{\infty}\left(\Gamma_{3}\right)\right)}=0$.

(a) the operator $\mathcal{B}_{\rho}: Q \times \mathbb{S}^{d} \longrightarrow \mathbb{S}^{d}$ satisfies (33) with $L_{\mathcal{B}_{\rho}}>0$ for each $\rho>0$ and $L_{\mathcal{B}_{\rho}} \leqslant L_{3}$ for each $\rho>0$,

(b) there exists $B: \mathbb{R}_{+} \longrightarrow \mathbb{R}_{+}$such that $\left\|\mathcal{B}_{\rho}(x, t, \varepsilon)-\mathcal{B}(x, t, \varepsilon)\right\|_{\mathbb{S}^{d}} \leqslant B(\rho)\|\varepsilon\|_{\mathbb{S}^{d}}$ for all $\varepsilon \in \mathbb{S}^{d}$, a.e. $(x, t) \in Q$, for each $\rho>0$ and $\lim _{\rho \rightarrow 0} B(\rho)=0$.

(a) the function $p_{\rho}: \Sigma_{3} \times \mathbb{R} \longrightarrow \mathbb{R}$ satisfies (35) with $L_{p_{\rho}}>0$ for each $\rho>0$ and $L_{p_{\rho}} \leqslant L_{4}$ for each $\rho>0$,

(b) there exists $P: \mathbb{R}_{+} \longrightarrow \mathbb{R}_{+}$such that

$\left|p_{\rho}(x, t, r)-p(x, t, r)\right| \leqslant P(\rho)|r|$ for all $r \in \mathbb{R}$ a.e. $(x, t) \in \Sigma_{3}$, for each $\rho>0$ and $\lim _{\rho \rightarrow 0} P(\rho)=0$.

$u_{0}, u_{0 \rho} \in V \quad$ and $\quad u_{0 \rho} \longrightarrow u_{0} \quad$ in $V$, as $\rho \longrightarrow 0$.

The perturbed version of Problem 17 is the following. Let $\rho>0$. 
Problem 20 Find a displacement field $u_{\rho} \in \mathcal{V}$ such that $u_{\rho}^{\prime} \in \mathcal{W}$ and

$$
\begin{aligned}
& \left\langle u_{\rho}^{\prime \prime}(t), v\right\rangle_{V^{*} \times V}+\left\langle\mathcal{A}_{\rho}\left(t, \varepsilon\left(u_{\rho}^{\prime}(t)\right)\right), \varepsilon(v)\right\rangle_{\mathcal{H}}+\left\langle\mathcal{B}_{\rho}\left(t, \varepsilon\left(u_{\rho}(t)\right)\right), \varepsilon(v)\right\rangle_{\mathcal{H}} \\
& +\left\langle\int_{0}^{t} \mathcal{C}_{\rho}(t-s) \varepsilon\left(u_{\rho}^{\prime}(s)\right) d s, \varepsilon(v)\right\rangle_{\mathcal{H}}+\int_{\Gamma_{3}} p_{\rho}\left(t, u_{\rho v}(t)\right) v_{\nu} d \Gamma \\
& \quad+\int_{\Gamma_{3}}\left(\int_{0}^{t} b_{\rho}(t-s) u_{\rho v}(s) d s\right) v_{\nu} d \Gamma \\
& \quad+\int_{\Gamma_{3}} F_{b}\left(\left\|u_{\rho \tau}(t)\right\|_{\mathbb{R}^{d}}\right) j_{\tau}^{0}\left(t, u_{\rho \tau}^{\prime}(t) ; v_{\tau}\right) d \Gamma \geqslant\left\langle f_{\rho}(t), v\right\rangle_{V^{*} \times V}
\end{aligned}
$$

for all $v \in V$, a.e. $t \in(0, T)$ and with $u_{\rho}(0)=u_{0 \rho}, u_{\rho}^{\prime}(0)=v_{0 \rho}$.

We have the following convergence result.

Theorem 21 Under assumptions (7), (8)(a), (14)(f), (g), (15), (37), (38), (47) and (59)-(62), the solution $u_{\rho}$ of Problem 20 converges to the solution $u$ of Problem 17, i.e.,

$$
\lim _{\rho \rightarrow 0}\left(\left\|u_{\rho}-u\right\|_{\mathcal{V}}+\left\|u_{\rho}^{\prime}-u^{\prime}\right\|_{\mathcal{V}}+\left\|u_{\rho}^{\prime}-u^{\prime}\right\|_{C(0, T ; H)}\right)=0
$$

Proof From Theorem 18, we deduce that, for every $\rho>0$, Problem 20 has a unique solution $u_{\rho} \in \mathcal{V}$ with $u_{\rho}^{\prime} \in \mathcal{W}$. We denote by $w_{\rho}=u_{\rho}^{\prime}$ the velocity field. For each $\rho>0$, we define the operator $\mathcal{R}_{1 \rho}: \mathcal{V} \longrightarrow \mathcal{V}$ by

$$
\left(\mathcal{R}_{1 \rho} v\right)(t)=\int_{0}^{t} v(s) d s+u_{0 \rho} \quad \text { for all } v \in \mathcal{V}, \text { a.e. } t \in[0, T] .
$$

With this notation, Problem 20 can be written in the following form.

Problem 22 Find a velocity field $w_{\rho} \in \mathcal{W}$ such that

$$
\begin{aligned}
& \left\langle w_{\rho}^{\prime}(t), v\right\rangle_{V^{*} \times V}+\left\langle\mathcal{A}_{\rho}\left(t, \varepsilon\left(w_{\rho}(t)\right)\right), \varepsilon(v)\right\rangle_{\mathcal{H}}+\left\langle\mathcal{B}_{\rho}\left(t, \varepsilon\left(\left(\mathcal{R}_{1 \rho} w_{\rho}\right)(t)\right)\right), \varepsilon(v)\right\rangle_{\mathcal{H}} \\
& +\left\langle\int_{0}^{t} \mathcal{C}_{\rho}(t-s) \varepsilon\left(w_{\rho}(s)\right) d s, \varepsilon(v)\right\rangle_{\mathcal{H}}+\int_{\Gamma_{3}} p_{\rho}\left(t,\left(\mathcal{R}_{1 \rho} w_{\rho}\right)_{v}(t)\right) v_{v} d \Gamma \\
& \quad+\int_{\Gamma_{3}}\left(\int_{0}^{t} b_{\rho}(t-s)\left(\mathcal{R}_{1 \rho} w_{\rho}\right)_{v}(s) d s\right) v_{v} d \Gamma \\
& \quad+\int_{\Gamma_{3}} F_{b}\left(\left\|\left(\mathcal{R}_{1 \rho} w_{\rho}\right)_{\tau}(t)\right\|_{\mathbb{R}^{d}}\right) j_{\tau}^{0}\left(t, w_{\rho \tau}(t) ; v_{\tau}\right) d \Gamma \geqslant\left\langle f_{\rho}(t), v\right\rangle_{V^{*} \times V}
\end{aligned}
$$

for all $v \in V$ and a.e. $t \in(0, T)$ with $w_{\rho}(0)=v_{0 \rho}$.

We apply Theorem 13 for Problem 22. Now, for every $\rho>0$, we introduce the operator $A_{\rho}:(0, T) \times V \longrightarrow V^{*}$ defined by

$$
\left\langle A_{\rho}(t, v), z\right\rangle_{V^{*} \times V}=\left\langle\mathcal{A}_{\rho}(t, \varepsilon(v)), \varepsilon(z)\right\rangle_{\mathcal{H}}
$$


for all $v, z \in V$ and a.e. $t \in(0, T)$. Using (59)(a), we have

$$
\left\|\mathcal{A}_{\rho}(x, t, \varepsilon(v))-\mathcal{A}(x, t, \varepsilon(v))\right\|_{\mathbb{S}^{d}}^{2} \longrightarrow 0, \quad \text { as } \rho \longrightarrow 0
$$

for all $v \in V$, a.e. $(x, t) \in Q$. From (32)(c), we deduce

$$
\left\|\mathcal{A}_{\rho}(x, t, \varepsilon(v))-\mathcal{A}(x, t, \varepsilon(v))\right\|_{\mathbb{S}^{d}}^{2} \leqslant 4\left(\bar{a}_{0}^{2}(x, t)+\bar{a}_{1}^{2}\|\varepsilon(v)\|_{\mathbb{S}^{d}}^{2}\right)
$$

for a.e. $(x, t) \in Q$. Hence, by the Lebesgue dominated convergence theorem, we obtain

$$
\begin{aligned}
& \left\|\mathcal{A}_{\rho}(x, t, \varepsilon(v))-\mathcal{A}(x, t, \varepsilon(v))\right\|_{\mathcal{H}}^{2}=\int_{\Omega}\left\|\mathcal{A}_{\rho}(x, t, \varepsilon(v))-\mathcal{A}(x, t, \varepsilon(v))\right\|_{\mathbb{S}^{d}}^{2} d x \longrightarrow 0, \\
& \quad \text { as } \rho \longrightarrow 0
\end{aligned}
$$

On the other hand, by Hölder inequality, we have

$$
\begin{aligned}
\left\langle A_{\rho}(t, v)-A(t, v), z\right\rangle_{V^{*} \times V} & =\int_{\Omega}\left(\mathcal{A}_{\rho}(x, t, \varepsilon(v))-\mathcal{A}(x, t, \varepsilon(v))\right): \varepsilon(z) d x \\
& \leqslant\left\|\mathcal{A}_{\rho}(x, t, \varepsilon(v))-\mathcal{A}(x, t, \varepsilon(v))\right\|_{\mathcal{H}}^{2}\|z\|_{V}
\end{aligned}
$$

for all $v, z \in V$, a.e. $t \in(0, T)$. Hence, we have $A_{\rho}(t, v) \longrightarrow A(t, v)$ for all $v \in V$, a.e. $t \in(0, T)$, which implies (14)(a).

Subsequently, for every $\rho>0$, we define the operator $\mathcal{R}_{\rho}: \mathcal{V} \longrightarrow \mathcal{V}$ by

$$
\left(\mathcal{R}_{\rho} v\right)(t)=\left(\mathcal{R}_{1 \rho} v\right)_{\tau}(t) \quad \text { for all } v \in \mathcal{V} \quad \text { and a.e. } \quad t \in(0, T)
$$

and the operator $\mathcal{S}_{\rho}: \mathcal{V} \longrightarrow \mathcal{V}^{*}$ by

$$
\begin{aligned}
\left\langle\left(\mathcal{S}_{\rho} v\right)(t), z\right\rangle_{V^{*} \times V}= & \left\langle\mathcal{B}_{\rho}\left(t, \varepsilon\left(\mathcal{R}_{1 \rho} v\right)(t)\right), \varepsilon(z)\right\rangle_{\mathcal{H}}+\left\langle\int_{0}^{t} \mathcal{C}_{\rho}(t-s) \varepsilon(v(s)) d s, \varepsilon(z)\right\rangle_{\mathcal{H}} \\
& +\int_{\Gamma_{3}} p_{\rho}\left(t,\left(\mathcal{R}_{1 \rho} v\right)_{\nu}(t)\right) z_{\nu} d \Gamma \\
& +\int_{\Gamma_{3}}\left(\int_{0}^{t} b_{\rho}(t-s)\left(\mathcal{R}_{1 \rho} v\right)_{v}(t) d s\right) z_{\nu} d \Gamma
\end{aligned}
$$

for all $v \in \mathcal{V}, z \in V$, a.e. $t \in(0, T)$.

Now, we show that the operators $\mathcal{R}_{\rho}$ and $\mathcal{S}_{\rho}$ satisfy hypotheses (14)(b)-(e). Using (67), we observe that

$$
\left\|\left(\mathcal{R}_{\rho} u_{\rho}\right)(t)-\left(\mathcal{R}_{\rho} u\right)(t)\right\|_{V} \leqslant c_{\mathrm{e}}\|\gamma\| \int_{0}^{t}\left\|u_{\rho}(s)-u(s)\right\|_{V} d s
$$

and

$$
\left\|\left(\mathcal{R}_{\rho} u\right)(t)-(\mathcal{R} u)(t)\right\|_{V} \leqslant c_{\mathrm{e}}\|\gamma\|\left\|u_{0 \rho}-u_{0}\right\|_{V}
$$

for a.e. $t \in(0, T)$. Hence, the conditions (14)(d) and (e) hold with $L_{\mathcal{R}_{\rho}}=c_{\mathrm{e}}\|\gamma\|, k_{2}(\rho)=$ $c_{\mathrm{e}}\|\gamma\|\left\|u_{0 \rho}-u_{0}\right\|_{V}$ and $g_{2}(u)=1$. Using (62), it easy to see that $k_{2}(\rho) \longrightarrow 0$, as $\rho \longrightarrow 0$. Hence (14)(d) and (e) hold. 
Note that, using (59)-(62) in (68), we obtain

$$
\begin{aligned}
\left\|\left(\mathcal{S}_{\rho} u_{\rho}\right)(t)-\left(\mathcal{S}_{\rho} u\right)(t)\right\|_{V^{*}} \leqslant & \left(L_{\mathcal{B}_{\rho}}+\left\|\mathcal{C}_{\rho}\right\|_{L^{\infty}\left(Q ; \mathbb{S}^{d}\right)}\right. \\
& \left.+c_{\mathrm{e}}^{2}\|\gamma\|^{2}\left(\left\|b_{\rho}\right\|_{L^{1}\left(0, T, L^{\infty}\left(\Gamma_{3}\right)\right)}+L_{p_{\rho}}\right)\right) \int_{0}^{t}\left\|u_{\rho}(s)-u(s)\right\|_{V} d s
\end{aligned}
$$

for a.e. $t \in(0, T)$. From this, (60)(a) and (61)(a), we obtain

$$
\begin{aligned}
\left\|\left(\mathcal{S}_{\rho} u_{\rho}\right)(t)-\left(\mathcal{S}_{\rho} u\right)(t)\right\|_{V^{*}} \leqslant & \left(L_{3}+\left\|\mathcal{C}_{\rho}\right\|_{L^{\infty}\left(Q ; \mathbb{S}^{d}\right)}\right. \\
& \left.+c_{\mathrm{e}}^{2}\|\gamma\|^{2}\left(\left\|b_{\rho}\right\|_{L^{1}\left(0, T, L^{\infty}\left(\Gamma_{3}\right)\right)}+L_{4}\right)\right) \int_{0}^{t}\left\|u_{\rho}(s)-u(s)\right\|_{V} d s
\end{aligned}
$$

for a.e. $t \in(0, T)$. From the above calculations, we deduce that (14)(b) holds with $L_{1}=L_{3}+$ $\left\|\mathcal{C}_{\rho}\right\|_{L^{\infty}\left(Q ; \mathbb{S}^{d}\right)}+c_{\mathrm{e}}\|\gamma\|^{2}\left(\left\|b_{\rho}\right\|_{L^{1}\left(0, T, L^{\infty}\left(\Gamma_{3}\right)\right)}+L_{4}\right)$. On the other hand from (60)(b), (61)(b) and (68), we have

$$
\begin{aligned}
& \left\|\left(\mathcal{S}_{\rho} u\right)(t)-(\mathcal{S} u)(t)\right\|_{V^{*}} \\
& \leqslant L_{3}\left\|u_{0 \rho}-u_{0}\right\|_{V}+B(\rho)\left\|\left(\mathcal{R}_{1} u\right)(t)\right\|_{V} \\
& \quad+\left\|\mathcal{C}_{\rho}-\mathcal{C}\right\|_{L^{\infty}\left(Q ; \mathbb{S}^{d}\right)} \int_{0}^{t}\|u(s)\|_{V} d s+L_{4}\|\gamma\|^{2} c_{\mathrm{e}}^{2}\left\|u_{0 \rho}-u_{0}\right\|_{V} \\
& \quad+P(\rho)\|\gamma\|^{2} c_{\mathrm{e}}^{2}\left\|\left(\mathcal{R}_{1} u\right)(t)\right\|_{V}+\left\|b_{\rho}\right\|_{L^{1}\left(0, T ; L^{2}\left(\Gamma_{3}\right)\right)} c_{\mathrm{e}}^{2}\|\gamma\|^{2}\left\|u_{0 \rho}-u_{0}\right\|_{V} \\
& \quad+\left\|b_{\rho}-b\right\|_{L^{1}\left(0, T ; L^{2}\left(\Gamma_{3}\right)\right)} c_{\mathrm{e}}^{2}\|\gamma\|^{2}\left\|\left(\mathcal{R}_{1} u\right)(t)\right\|_{V}
\end{aligned}
$$

for a.e. $t \in(0, T)$. Hence, we see that the condition (14)(c) is satisfied with

$$
\begin{aligned}
k_{1}(\rho)= & L_{3}\left\|u_{0 \rho}-u_{0}\right\|_{V}+B(\rho)+\left\|\mathcal{C}_{\rho}-\mathcal{C}\right\|_{L^{\infty}\left(Q ; \mathbb{S}^{d}\right)}+L_{4}\|\gamma\|^{2} c_{\mathrm{e}}^{2}\left\|u_{0 \rho}-u_{0}\right\|_{V} \\
& +P(\rho)\|\gamma\|^{2} c_{\mathrm{e}}^{2}+\left\|b_{\rho}\right\|_{L^{1}\left(0, T ; L^{2}\left(\Gamma_{3}\right)\right)} c_{\mathrm{e}}^{2}\|\gamma\|^{2}\left\|u_{0 \rho}-u_{0}\right\|_{V} \\
& +\left\|b_{\rho}-b\right\|_{L^{1}\left(0, T ; L^{2}\left(\Gamma_{3}\right)\right)} c_{\mathrm{e}}^{2}\|\gamma\|^{2}
\end{aligned}
$$

and $g_{1}(u)=\max \left\{1, \int_{0}^{t}\|u(s)\|_{V} d s,\left\|\left(\mathcal{R}_{1} u\right)(t)\right\|_{V}\right\}$. Using conditions (59)(b) and (c), (60)(b), (61)(b) and (62), we have $k_{1}(\rho) \longrightarrow 0$, as $\rho \longrightarrow 0$.

Finally, we define the functional $J:(0, T) \times L^{2}\left(\Gamma_{3} ; \mathbb{R}^{d}\right)^{2} \longrightarrow \mathbb{R}$ as in (54). This functional satisfies (56) and (57). Using (66)-(68) in Problem 22, and a reasoning analogous as in the proof of Theorem 18, we associate with Problem 22, the following subdifferential inclusion.

Problem 23 Find $w_{\rho} \in \mathcal{W}$ such that

$$
\begin{aligned}
& w_{\rho}^{\prime}(t)+A_{\rho}\left(t, w_{\rho}(t)\right)+\left(\mathcal{S}_{\rho} w_{\rho}\right)(t)+F\left(t,\left(\mathcal{R}_{\rho} w_{\rho}\right)(t), w_{\rho}(t)\right) \ni f_{\rho}(t) \\
& \quad \text { for a.e. } t \in(0, T) \\
& w_{\rho}(0)=v_{0 \rho} .
\end{aligned}
$$

Based on Theorem 13, we deduce that

$$
\left\|w_{\rho}-w\right\|_{\mathcal{V}}+\left\|w_{\rho}-w\right\|_{C(0, T ; H)} \longrightarrow 0, \quad \text { as } \rho \longrightarrow 0,
$$


where $w, w_{\rho} \in \mathcal{W}$ are the unique solutions to Problem 58 and Problem 12, respectively. Hence and from the fact that $u_{\rho}^{\prime}(t)=w_{\rho}(t)$ and $u^{\prime}(t)=w(t)$, we have

$$
\lim _{\rho \rightarrow 0}\left(\left\|u_{\rho}^{\prime}-u^{\prime}\right\|_{\mathcal{V}}+\left\|u_{\rho}^{\prime}-u^{\prime}\right\|_{C(0, T ; H)}\right)=0
$$

Note that from (48), we obtain $\left\|u_{\rho}-u\right\|_{\mathcal{V}} \leqslant \sqrt{2} T\left\|u_{\rho}^{\prime}-u\right\|_{\mathcal{V}}+\sqrt{2 T}\left\|u_{0 \rho}-u_{0}\right\|_{V}$. This inequality combined with (62) and (71) implies that

$$
\lim _{\rho \rightarrow 0}\left\|u_{\rho}-u\right\|_{\mathcal{V}}=0
$$

This completes the proof of the theorem.

In addition to the mathematical interest in the convergence results (71) and (72), they are of importance from the mechanical point of view, since they provide a dependence of the weak solution of the Problem 14 on the viscosity operator, elasticity operator, relaxation operator, normal compliance function, the surface memory function, as well. Moreover, it is clear that if $f_{0 \rho} \longrightarrow f_{0}$ in $L^{2}\left(0, T ; L^{2}\left(\Omega ; \mathbb{R}^{d}\right)\right)$ and $f_{2 \rho} \longrightarrow f_{2}$ in $L^{2}\left(0, T ; L^{2}\left(\Gamma_{2} ; \mathbb{R}^{d}\right)\right)$, as $\rho \longrightarrow 0$, then from the definition (45), we obtain that $f_{\rho} \longrightarrow f$ in $\mathcal{V}$, as $\rho \longrightarrow 0$.

We conclude this paper with a simple example of a normal compliance function for which the condition (61) holds. Let $c_{\nu \rho}, c_{v}>0$ be such that $c_{v \rho} \longrightarrow c_{v}$, as $\rho \longrightarrow 0$. Assume that the functions $p$ and $p_{v}$ are defined by

$$
p(r)=c_{\nu} r_{+}, \quad p_{\rho}(r)=c_{\nu \rho} r_{+} \quad \text { for all } r \in \mathbb{R},
$$

where $r_{+}=\max \{0, r\}$ and $c_{\nu}, c_{\nu \rho}$ represent the surface stiffness coefficients. We remark that the dependence of these functions on stiffness coefficients is important in contact problems. When $c_{v}$ is larger the reaction force of the foundation to penetration is larger and so the same force will result in a smaller penetration, which means that the foundation is less deformable. When $c_{v}$ is smaller the reaction force of the foundation to penetration is smaller, and then the foundation is less stiff and more deformable. For more discussion, we refer to (see Chap. 4 in [27]). It easy to see that $\left|p_{\rho}(r)-p(r)\right| \leqslant\left|c_{\nu \rho}-c_{\nu}\right||r| \leqslant P(\rho)|r|$ for all $r \in \mathbb{R}$ with $P(\rho)=\left|c_{v \rho}-c_{v}\right| \longrightarrow 0$, as $\rho \longrightarrow 0$. Since the function $r \rightarrow r_{+}$is Lipschitz continuous, we conclude that the condition (61) is satisfied.

Open Access This article is distributed under the terms of the Creative Commons Attribution 4.0 International License (http://creativecommons.org/licenses/by/4.0/), which permits unrestricted use, distribution, and reproduction in any medium, provided you give appropriate credit to the original author(s) and the source, provide a link to the Creative Commons license, and indicate if changes were made.

\section{References}

1. Barboteu, M., Bartosz, K., Kalita, P., Ramadan, A.: Analysis of a contact problem with normal compliance, finite penetration and nonmonotone slip dependent friction. Commun. Contemp. Math. 16, 1-29 (2014)

2. Barboteu, M., Danan, D., Sofonea, M.: Analysis of a contact problem with normal damped response and unilateral constraint. Z. Angew. Math. Mech. (2015). doi:10.1002/zamm.201400304

3. Chau, O., Fernandez-Garcia, J.R., Han, W., Sofonea, M.: A frictionless contact problem for elasticviscoplastic materials with normal compliance and damage. Comput. Methods Appl. Mech. Eng. 191, 5007-5026 (2002)

4. Chau, O., Shillor, M., Sofonea, M.: Dynamic frictionless contact with adhesion. Z. Angew. Math. Phys. 1, 32-47 (2004) 
5. Cheng, X., Migórski, S., Ochal, A., Sofonea, S.: Analysis of two quasistatic history-dependent contact models. Discrete Contin. Dyn. Syst., Ser. B 8(19), 2425-2445 (2014)

6. Clarke, F.H.: Optimization and Nonsmooth Analysis. Canad. Math. Soc. Ser. Monogr. Adv. Texts. Wiley, New York (1983)

7. Denkowski, Z., Migórski, S., Papageorgiou, N.S.: An Introduction to Nonlinear Analysis: Applications. Kluwer Academic/Plenum, Boston, Dordrecht/London, New York (2003)

8. Farcas, A., Patrolescu, F., Sofonea, M.: A history-dependent contact problem with unilateral constrain. Ann. Acad. Rom. Sci. Ser. Math. Appl. 4(1), 90-96 (2012)

9. Fernandez, J.R., Santamarina, D.: A dynamic viscoelastic contact problem with normal compliance. J. Comput. Appl. Math. 276, 30-46 (2015)

10. Ionescu, I.R., Nguyen, Q.: Dynamic contact problem with slip-dependent friction in viscoelasticity. Int. J. Appl. Math. Comput. Sci. 12, 71-80 (2002)

11. Ionescu, I.R., Nguyen, Q., Wolf, S.: Slip-dependent friction in dynamic elasticity. Nonlinear Anal. 53, 375-390 (2003)

12. Ionescu, I.R., Paumier, J.: On the contact problem with slip displacement dependent friction in elastostatics. Int. J. Eng. Sci. 34, 471-491 (1996)

13. Klarbring, A., Mikelic, A., Shillor, M.: Frictional contact problems with normal compliance. Int. J. Eng. Sci. 26, 811-832 (1988)

14. Klarbring, A., Mikelic, A., Shillor, M.: On friction problems with normal compliance. Nonlinear Anal. 13, 935-955 (1989)

15. Kulig, A., Migórski, S.: Solvability and continuous dependence results for second order nonlinear evolution inclusion with a Volterra-type operator. Nonlinear Anal. 75, 4729-4746 (2012)

16. Migórski, S.: Evolution hemivariational inequality for a class of dynamic viscoelastic nonmonotone frictional contact problems. Comput. Math. Appl. 52, 677-698 (2006)

17. Migórski, S.: A class of hemivariational inequalities for electroelastic contact problems with slip dependent friction. Discrete Contin. Dyn. Syst., Ser. S 1(1), 117-126 (2008)

18. Migórski, S., Ochal, A.: Hemivariational inequality for viscoelastic contact problem with slip-dependent friction. Nonlinear Anal. 61, 135-161 (2005)

19. Migórski, S., Ochal, A., Sofonea, S.: History-dependent subdifferential inclusions and hemivariational inequality in contact mechanics. Nonlinear Anal., Real World Appl. 12, 3385-3396 (2011)

20. Migórski, S., Ochal, A., Sofonea, M.: History-dependent hemivariational inequalities with applications to Contact Mechanics. Ann. Univ. Buchar. Math. Ser. 4(LXII), 193-212 (2013)

21. Migórski, S., Ochal, A., Sofonea, M.: Nonlinear Inclusions and Hemivariational Inequalities. Models and Analysis of Contact Problems. Advances in Mechanics and Mathematics, vol. 26. Springer, New York (2013)

22. Oden, J.T., Martins, J.A.C.: Models and computational methods for dynamic friction phenomena. Comput. Methods Appl. Mech. Eng. 52, 527-634 (1985)

23. Ogorzały, J.: Quasistatic bilateral contact problem with time delay for viscoelastic materials. Math. Mech. Solids (2014). doi:10.1177/1081286514552208

24. Rabinowicz, E.: The nature of the static and kinetic coefficients of friction. J. Appl. Phys. 22(11), 1373$1379(1951)$

25. Sofonea, M., Han, W., Barboteu, M.: Analysis of a viscoelastic contact problem with multivalued normal compliance and unilateral constraint. Comput. Methods Appl. Mech. Eng. 264, 12-22 (2013)

26. Sofonea, M., Farcas, A.: Analysis of a history-dependent frictional contact problem. Appl. Anal. 2, 428444 (2014)

27. Sofonea, M., Matei, A.: Mathematical Models in Contact Mechanics. London Mathematical Society Lecture Note Series, vol. 398. Cambridge University Press, Cambridge (2012)

28. Sofonea, M., Patrulescu, F.: Analysis of a history-dependent frictionless contact problems. Math. Mech. Solids 18(4), 409-430 (2012)

29. Sofonea, M., Patrulescu, F., Farcas, A.: A Viscoplastic contact problem with normal compliance, unilateral constraint and memory term. Appl. Math. Optim. 69, 175-198 (2014)

30. Sofonea, M., Shillor, M., Telega, J.: Models and Analysis of Quasistatic Contact. Variational Methods. Springer, Berlin (2004)

31. Yao, S., Huang, N.: A quasistatic contact problem for viscoelastic materials with slip-dependent friction and time delay. Math. Probl. Eng. 2012, 1-23 (2012)

32. Zeidler, E.: Nonlinear Functional Analysis and Applications, II A/B. Springer, New York (1999)

33. Zhu, S.W.: Optimal control of variational inequalities with delays in the highest order spatial derivatives. Acta Math. Sin. Engl. Ser. 22, 607-624 (2006) 